\title{
Die Migrationspolitik der deutschen Länder
}

\author{
Eine mehrdimensionale Analyse
}

\author{
Daniel Meyer $\cdot$ Jonas Philipp $\cdot$ Georg Wenzelburger
}

Eingegangen: 16. April 2020 / Überarbeitet: 10. Dezember 2020 / Angenommen: 19. Dezember 2020 /

Online publiziert: 22. Januar 2021

(C) Der/die Autor(en) 2021

Zusammenfassung Der vorliegende Aufsatz untersucht, (1) inwieweit Unterschiede in der Ausgestaltung der Migrationspolitik auf substaatlicher Ebene in der Bundesrepublik Deutschland bestehen und (2) wodurch sich die Policy-Varianz zwischen den deutschen Ländern erklären lässt. Während bestehende Studien ähnlich gelagerte Fragen meist nur auf Basis eines spezifischen Indikators der Migrationspolitik untersucht haben - wie etwa der Ausgaben - schlagen wir ein mehrdimensionales Messkonzept vor, das sechs unterschiedliche Dimensionen der Migrationspolitik auf Länderebene unterscheidet: (1) die Art der Unterbringung, (2) die Art der Leistungserbringung, (3) die Gesundheitsversorgung, (4) die Aufnahmepraxis, (5) die Abschiebepraxis, sowie die (6) bundesstaatliche Positionierung am Beispiel der „,sicheren Herkunftsländer“. Zur Analyse möglicher Pfade zur Erklärung der Unterschiede zwischen den Bundesländern nutzen wir eine fuzzy-set QCA-Analyse und greifen auf Parteipolitik, sozioökonomischen Kontext und die Einstellungen der Bevölkerung als Bedingungen zurück.

Unsere Ergebnisse zeigen, dass in der Tat substanzielle Unterschiede zwischen den Bundesländern bestehen. Zudem finden wir, dass die parteipolitische Zusammensetzung der Regierung in unterschiedlichen Pfaden eine wichtige Bedingung für das Vorliegen restriktiver bzw. permissiver Migrationspolitik ist. In keinem einzigen kausalen Pfad der fsQCA-Analyse ist überhaupt eine Erklärung restriktiver bzw. permissiver Migrationspolitik ohne Berücksichtigung der Parteiideologie möglich - ein Ergebnis, das klar für die hohe Relevanz der parteipolitischen Zusammensetzung der Regierung spricht. Die Einstellungsmuster der Bevölkerung in dem jeweiligen Bundesland, die Migrationspolitik und die sozioökonomischen Bedingungen scheinen hingegen nur eine untergeordnete Rolle zu spielen.

Prof. Dr. G. Wenzelburger $(\bowtie)$

Professur für Policy-Analyse und Politische Ökonomie, TU Kaiserslautern, Postfach

3049, 67663 Kaiserslautern, Deutschland

E-Mail: georg.wenzelburger@sowi.uni-kl.de 
Schlüsselwörter Migrationspolitik · Bundesländervergleich · Mehrebenensystem · Deutschland · fsQCA

\title{
Migration policies in the German Länder
}

A multi-dimensional analysis

\begin{abstract}
This paper examines (1) the extent to which migration policies vary at the subnational level in the Federal Republic of Germany and (2) how the policy variance between the German Länder can be explained. Going beyond existing work, we propose a multidimensional measurement concept that distinguishes six different dimensions of migration policy at the state level: (1) the type of accommodation, (2) the type of services provided, (3) health care, (4) admission practices, (5) deportation practices, and (6) state positioning in the context of "safe countries of origin". Moreover, we analyze possible paths to explain the differences between the states, using a fuzzy-set QCA analysis and draw on a theoretical framework that combines party politics, socioeconomic context, and the attitudes of the population as conditions.

Our results show that there are substantial differences between the federal states. We also find that the party political composition of the government in different paths is an important condition for the existence of restrictive or permissive migration policies. In not a single causal path of the fsQCA analysis is it possible to explain restrictive or permissive migration policy without taking party ideology into account - a result that clearly speaks for the high relevance of the party political composition of the government. In contrast, the attitudes of the population in each state, migration policy and socioeconomic conditions seem to play only a minor role.
\end{abstract}

Keywords Migration policy · Federal states · Multi-level governance · Germany · fsQCA

\section{Einleitung}

Spätestens, seitdem Geflüchtete aus dem globalen Süden Zuflucht in den europäischen Ländern - und allen voran in Deutschland - gesucht haben, ist die Migrationspolitik verstärkt in den Fokus politikwissenschaftlicher Analysen gerückt. Dabei stand sowohl die europäische Dimension der Migrationspolitik - etwa die (Un-)Möglichkeit einer solidarischer Verteilung der Migranten auf die EU-Staaten im Zentrum der Debatte (de Wenden 2017; Geddes 2018) als auch die Frage, welche nationalstaatlich unterschiedlichen Wege in der Migrationspolitik sichtbar sind und wie sich diese erklären lassen (Consterdine und Hampshire 2019; Helbling und Kalkum 2018). In Mehrebenensystemen wie der Bundesrepublik Deutschland stellt sich darüber hinaus jedoch die Frage, wie die auf europäischer oder nationaler Ebene beschlossenen Policies auf den unteren Ebenen des Staates umgesetzt werden und in welchem Ausmaß sich auch hier Unterschiede in der Ausgestaltung der Migra- 
tionspolitik auf substaatlicher Ebene zeigen bzw. wodurch sich die Policy-Varianz zwischen den Gliedstaaten föderaler politischer Systeme erklären lässt.

Dieser Aufsatz nimmt daher die Ebene der deutschen Länder in den Blick und untersucht dabei zwei zentrale Forschungsfragen. Die erste Frage ist deskriptiver Natur und analysiert, inwiefern die Bundesländer unterschiedliche migrationspolitische Wege beschritten haben. Wir schlagen dabei ein Messkonzept vor, das sechs unterschiedliche Dimensionen der Migrationspolitik auf Länderebene unterscheidet: (1) die Art der Unterbringung, (2) die Art der Leistungserbringung, (3) die Gesundheitsversorgung, (4) die Aufnahmepraxis, (5) die Abschiebepraxis, sowie die (6) bundesstaatliche Positionierung am Beispiel der „sicheren Herkunftsländer“.

In diesen sechs Dimensionen verfügen die Bundesländer über eigenen Handlungsspielraum, weshalb dort Varianz sichtbar werden kann. Mit dieser umfassenden Vermessung der Migrationspolitik gehen wir erheblich über den bisherigen Forschungsstand hinaus. Denn bisher wurden vor allem einzelne Aspekte der Migrationspolitik analysiert - z. B. Henkes (2008) und, sehr früh, Thränhardt (2001) zu Einbürgerungsquoten, Riedel und Schneider (2017) zu Ablehnungsquoten, Hörisch und Heiken zu dezentraler Unterbringung, Wolf (2011) zu Härtefallanträgen, Hörisch (2018) zur Ausgabenhöhe und zur Abschiebebereitschaft nach Afghanistan, oder Günther et al. (2019) zur Ausgestaltung der Gesundheitsversorgung. Die Studie, welche unserem Vorgehen bei der Vermessung der Asylpolitik am ehesten ähnelt, ist die Analyse von Reiter und Töller (2019), die ebenfalls sechs Betrachtungseinheiten aufnehmen. Allerdings geht unser Messkonzept über die genannte Untersuchung hinaus, da in einzelnen Aspekten eine feingliedrigere Messung möglich ist, als sie von Reiter und Töller vorgenommen wurde (s. unten).

Daneben geht unsere Analyse auch die Frage an, wie die sichtbaren Unterschiede zwischen den Bundesländern erklärt werden können und führt dazu eine fuzzy-set QCA-Analyse (fsQCA) durch. Ein ähnliches Vorgehen wählt etwa Hörisch (2018) er beschränkt sich jedoch auf die Asylausgaben der Länder und deren Abschiebebereitschaft nach Afghanistan - während Reiter und Töller (2019) auf Basis ihrer umfassenden Vermessung keine weiterführende Analyse zu möglichen Erklärungen der sichtbaren Unterschiede zwischen den Bundesländern vornehmen. ${ }^{1}$ Hinsichtlich der Erklärungen für Unterschiede in der Migrationspolitik zwischen den deutschen Ländern finden einige Studien Evidenz für eine Beeinflussung der Migrationspolitik durch die parteipolitischen Ausrichtungen der amtierenden Landesregierungen (etwa: Hörisch 2018; Günther et al. 2019), während andere Autoren keinen Zusammenhang sehen (Reiter und Töller 2019, S. 214; Sack und Töller 2018, S. 608). Daneben scheint ein gewisser Unterschied zwischen den Ländern im Westen und im Osten der Republik zu bestehen (Hörisch 2018). Sehr uneinheitlich sind hingegen die Befunde zum Einfluss sozio-demographischer oder wirtschaftlicher Faktoren hier scheinen die Ergebnisse je nach betrachtetem Detailaspekt der Migrationspolitik

\footnotetext{
1 Dies sind: Anteil dezentraler Unterbringung, Beauftragung von Krankenkassen/Gesundheitskarte; Abschiebung nach Afghanistan; Rückführungsquote; Anteil von Abschiebungen an Rückführungen; Errichtung von Ankerzentren.
} 
voneinander abzuweichen (siehe dazu etwa Hörisch 2018; Wolf 2011; oder Riedel und Schneider 2017). ${ }^{2}$

Unsere Ergebnisse zeigen, dass in der Tat substanzielle Unterschiede zwischen den Bundesländern bestehen und die Aufnahme weiterer Dimensionen der Migrationspolitik eine differenziertere Betrachtung des Untersuchungsgegenstandes ermöglicht. Auf Basis der auf diese Weise vermessenen Migrationspolitik finden wir, dass die parteipolitische Zusammensetzung der Regierung eine zentrale Bedingung für das Vorliegen restriktiver bzw. permissiver Migrationspolitik ist: Wenn Parteien mit einer klar konservativ-autoritären Parteiprogrammatik an der Regierung beteiligt sind, ist die Migrationspolitik häufig restriktiv, während umgekehrt Landesregierungen mit Beteiligung von Parteien mit liberaler Programmatik (z. B. Grüne, FDP) häufig mit permissiven Policies einhergehen. In keinem einzigen kausalen Pfad der fsQCA-Analyse ist überhaupt eine Erklärung restriktiver bzw. permissiver Migrationspolitik ohne Berücksichtigung der Parteiideologie möglich, was auf die zentrale Rolle von politischen Parteien in der Kette der Responsivität hindeutet (dazu: Wenzelburger et al. 2020).

Der Aufsatz ist in fünf Abschnitte gegliedert. Im nächsten Abschnitt stellen wir unser Konzept für die Vermessung der Migrationspolitik auf Ebene der deutschen Länder vor. Dabei grenzen wir unseren Ansatz von bestehenden Konzepten ab und zeigen, an welchen Stellen wir mehr Informationen zur Policy-Varianz verarbeiten als bisherige Studien. Darauf aufbauend zeigen wir, welches Bild sich ergibt, wenn man die Migrationspolitik anhand der von uns vorgeschlagenen sechs Subdimensionen betrachtet. Im dritten Abschnitt stellen wir kurz die theoretische Grundlage für die Analyse der Varianz zwischen den Bundesländern vor und formulieren konkrete Hypothesen. Wir diskutieren auch die gewählte Methode und die Kalibrierung der Bedingungen. Die empirische Analyse der Bedingungskonstellationen erfolgt in Abschn. 4. Der letzte Teil des Beitrags fasst die empirischen Ergebnisse in einem Fazit zusammen und diskutiert diese im Kontext des Forschungsstandes.

\section{Migrationspolitik im Bundesländervergleich: Messkonzept und Policy-Muster}

Um migrationspolitische Unterschiede zwischen den Bundesländern freizulegen und zu erklären, geht dieser Aufsatz über den bisherigen Forschungsstand in zwei zentralen Aspekten hinaus. Zum ersten beschränken wir uns nicht auf einzelne Aspekte der Migrationspolitik und analysieren diese getrennt voneinander (Riedel und Schneider 2017; Hörisch 2018; Günther et al. 2019), sondern aggregieren die unterschiedlichen Instrumente, mit denen Landesregierungen migrationspolitisch steuern können, zu einem übergreifenden Indikator (ähnlich auch: Reiter und Töller 2019). Zum zweiten - und im Gegensatz zu Reiter und Töller (2019) - verbleiben wir jedoch nicht

\footnotetext{
2 Ein Grund hierfür könnte auch die enge Verschränkung mit der kommunalen Ebene sein, die in unterschiedlichem Maße von den Ländern einbezogen wird (dazu etwa der Sammelband von Bogumil et al. 2019; und insbesondere Radtke und Hustedt 2019).
} 
bei der Beschreibung der so herausgearbeiteten migrationspolitischen Unterschiede zwischen den Ländern, sondern erklären diese mithilfe einer fsQCA.

Zur Messung der Policy-Varianz greifen wir auf sechs Dimensionen zurück: (1) die Art der Unterbringung, (2) die Art der Leistungserbringung, (3) die Gesundheitsversorgung, (4) die Aufnahmepraxis, (5) die Abschiebepraxis, sowie die (6) bundesstaatliche Positionierung am Beispiel der „sicheren Herkunftsländer“. Die Wahl dieser Dimensionen basiert auf zwei Erwägungen. Zum einen gilt es die Kompetenzverteilung in der Migrationspolitik im deutschen Mehrebenensystem zu berücksichtigen. Denn obwohl der Bund z. B. im Asylrecht den Rahmen festlegt, verfügen die Länder durchaus über eigene Spielräume (Schammann 2019). So ist der Bund zwar grundsätzlich im Rahmen der konkurrierenden Gesetzgebung etwa für die Erstaufnahme von Geflüchteten und Fragen des Niederlassungsrechts zuständig (Art. 74, GG) - und hat dies auch über das BAMF behördlich geregelt. Nach Asylbewerberleistungsgesetz haben jedoch die Länder weitreichende Kompetenzen in der Versorgung und Integration der Migranten (Behnke 2019, S. 67-70). Fokussiert man die Unterschiede zwischen den Ländern, sind daher insbesondere diese Dimensionen relevant. Zum zweiten sollte die in den Dimensionen gemessene Varianz auf Politikinstrumente zurückzuführen sein, die landespolitisch entschieden werden können und wo daher auch ein landespolitisches Erklärungsmodell ansetzen kann. Daher wurden etwa Sprach- und Integrationskurse nicht aufgenommen, weil deren Bereitstellung stark durch die kommunale Ebene beeinflusst wird und daher trotz bestehender Varianz zwischen den Ländern ein Erklärungsmodell auch die kommunale Ebene einbeziehen müsste (für einen Überblick: Hummel und Thöne 2016). Aus einem ähnlichen Grund haben wir uns dagegen entschieden, die Ausgaben für Asylpolitik, die etwa Hörisch (2018) als abhängige Variable nutzt, in das Messkonzept aufzunehmen. Denn zum einen bleibt der Zusammenhang zwischen Ausgabenhöhe und Restrikitivität von Asylpolitik unklar - hohe Ausgaben können durch permissive und restriktive Maßnahmen entstehen; Und zum anderen führt die länderspezifisch unterschiedlich ausgestaltete Aufgabenteilung mit der kommunalen Ebene dazu, dass der Indikator der Ausgaben nur schwer interpretierbar ist. Die Wahl der sechs Dimensionen zielt daher darauf ab, Varianz bei denjenigen politischen Instrumenten zu erheben, in denen Landespolitik tatsächlich für die Policy-Varianz entscheidend wird. Im Folgenden diskutieren wir knapp die einzelnen Dimensionen und beschreiben, wie wir diese zu einem übergreifenden Indikator zusammenführen.

\subsection{Art der Unterbringung von Geflüchteten}

Geflüchtete können auf zwei Arten untergebracht werden: entweder dezentral oder in Gemeinschaftsunterkünften. Die Bundesländer werden in $\S 44$ Abs. 1 AsylbLG verpflichtet, ,für die Unterbringung Asylbegehrender die dazu erforderlichen Aufnahmeeinrichtungen zu schaffen und zu unterhalten sowie entsprechend ihrer Aufnahmequote die im Hinblick auf den monatlichen Zugang Asylbegehrender in den Aufnahmeeinrichtungen notwendige Zahl von Unterbringungsplätzen bereitzustellen“. Um die Varianz bei der Art der Aufnahme und Unterbringungsverwaltung zwischen den Bundesländern genauer zu erfassen, wird der Anteil der dezentralen Unterbringung eines Bundeslandes erfasst und als ein Parameter für eine restrik- 
tive oder permissive Migrationspolitik herangezogen (Hörisch und Heiken 2018). Permissiv wäre in diesem Fall, wenn das Bundesland einen hohen Anteil an Dezentralisierung aufweist. Als restriktiv zu deuten sind „unvermeidbar schnell aus dem Boden gestampfte Massenunterbringungen [...], die weder menschenrechtlich vertretbar noch in ökonomischer Hinsicht langfristig vernünftig sind" (Wendel 2014, S. 7). So beschreibt beispielsweise Alexander Handschuh, Sprecher des deutschen Städte- und Gemeindebundes, dass eine Unterbringung in Gemeinschaftsunterkünften die Integration von Flüchtlingen erschwere (Stukenberg 2017).

In der Tat besteht erhebliche Varianz zwischen den Bundesländern bei dem Anteil der dezentralen Unterbringung (vgl. Tab. 1). Auffallend ist der Unterschied zwischen den beiden Bundesländern Schleswig-Holstein und Mecklenburg-Vorpommern. Das westliche norddeutsche Bundesland Schleswig-Holstein weist mit einem Anteil von 90,6\% der dezentralen Unterbringung den höchsten Wert im Bundesländervergleich auf, wohingegen das östliche Nachbarbundesland Mecklenburg-Vorpommern mit 29,5\% das Schlusslicht bildet. Der Mittelwert des prozentualen Anteils der dezentralen Unterbringung liegt bei $54,88 \%$.

\subsection{Existenzsicherung von Geflüchteten - Geld- oder Sachleistungen?}

Die finanzielle Verantwortung für Asylsuchende liegt im Kompetenzbereich der Bundesländer (Reiter und Töller 2019, S. 204) und umfasst insbesondere Grundleistungen nach dem Asylbewerberleistungsgesetz. Hierunter fällt zum einen der notwendige Bedarf nach § 3 AsylbLG - Ernährung, Unterkunft, Heizung, Kleidung, Gesundheitspflege und Gebrauchs- und Verbrauchsgüter eines Haushalts - sowie der notwendige persönliche Bedarf. Während der Spielraum für die Länder durch die bundeseinheitliche gesetzliche Regelung im AsylbLG sehr begrenzt ist - der notwendige persönliche Bedarf für einen alleinstehenden Leistungsberechtigten liegt z.B. nach $\S 3$ Abs. 1 AsylbLG bei $135 €^{3}$ - bestehen gewisse Abweichungsmöglichkeiten bei der Frage, ob eine Leistung als Sachleistung oder als Geldleistung bzw. als Wertgutschein ausgezahlt wird ( 3 Absatz 1 AsylbLG). So können Bundesländer bei „vertretbarem Verwaltungsaufwand“ auch Wertgutscheine ausstellen, wobei Umsetzungsspielraum besteht: Gerade den persönlich notwendigen Bedarf als Sachleistung zu vergeben, scheint in der Praxis schwierig, da zunächst einmal entschieden werden muss, was ein Geflüchteter überhaupt braucht. Zudem bedarf es Personal, welches einerseits Sachleistungen kauft und andererseits Wertgutscheine ausstellt, was zusätzliche Personalkosten erzeugt, die bei Geldleistungen nicht entstehen. Dieser Verwaltungsaufwand wird laut Kaiser (2015) von nahezu jedem Bundesland als zu hoch eingestuft. Lediglich Bayern versucht das Prinzip „Kein Bargeld an Flüchtlinge“" umzusetzen, wobei allerdings - nach den Daten von Gerl und Rehm (2016) - lediglich eine von acht Erstaufnahmeeinrichtungen (Donauwörth) dem Sachleistungsprinzip folgt. Da die Einführung von Sachleistungen und die damit verbundenen Komplikationen durch Wertgutscheine die freie Entfaltung eines Geflüchteten einschränkt, kann in diesem Zuge im Bundesland Bayern von einer restriktiven Migrationspolitik gesprochen werden. Alle anderen Bundesländer

\footnotetext{
${ }^{3}$ Eine genaue Auflistung für unterschiedliche Personengruppen ist aus $\S 3$ AsylbLG zu entnehmen.
} 
können als permissiv eingestuft werden, wobei der Bremer Senat bei seiner Begründung am weitesten geht und sich bei seiner Entscheidung pro Geldleistung auf das Urteil des Bundesverfassungsgerichts vom 18.07.2012 bezieht, wonach die Auszahlung des Barbetrags als grundlegendes Menschenrecht angesehen werden kann, welches nicht mit Sachleistungen zu gewährleisten sei (Kaiser 2015).

\subsection{Die Gesundheitsversorgung von Geflüchteten}

Einen Anspruch auf gesundheitliche Versorgung haben, laut $\S 1$ AsylbLG, alle Asylsuchenden, Bürgerkriegsflüchtlinge, Personen mit Duldung, vollziehbar ausreisepflichtige Personen sowie deren Familienangehörige. Leistungen in Einzelfällen - etwa bei stationären Aufenthalten, Krankheit, Schwangerschaft oder Geburt - sind ebenfalls geregelt (in $\S 4$ ), zudem können Psychotherapien für traumatisierte Asylsuchende als sonstige Leistungen aus $\S 6$ beansprucht werden (Reiter und Töller 2019, S. 203; Schammann 2015, S. 175). Die Bundesländer sind zuständig, wenn sich Geflüchtete noch in den Erstaufnahme- oder Nebeneinrichtungen des Landes befinden und keinen Asylantrag stellen konnten. Ebenso liegt die Eingangsuntersuchung im Kompetenzbereich des Bundeslandes (Wächter-Raquet 2016, S. 10). Besondere Regelungen liegen laut GKV-Spitzenverband (2016) bei der Gesundheitsversorgung von unbegleiteten minderjährigen Geflüchteten vor: Jugendämter werden gemäß $\S 42$ Abs. 2 Sa. 3 SGB VIII verpflichtet, ein unbegleitetes geflüchtetes Kind in Obhut zu nehmen sowie den notwendigen Unterhalt und die Krankenhilfe zu gewährleisten. Auch diese Gewährleistung unterscheidet sich von Bundesland zu Bundesland (GKV-Spitzenverband 2016). Als Vorreiter in der Gesundheitsversorgung von Geflüchteten ist der Stadtstaat Bremen zu nennen. Der Bremer Ansatz zielt darauf ab, Geflüchteten in Gemeinschaftsunterkünften eine Basisversorgung und ein Minimum an humanitärer Nothilfe zu gewährleisten. Somit erweitert das Bremer Modell, welches bereits 1990 in Bremen eingeführt wurde, die Zugangschancen von Geflüchteten im Gesundheitssystem. Darüber hinaus wurde bereits im Jahr 2005 eine elektronische Gesundheitskarte eingeführt, um bürokratische Hürden zu verringern.

Durch das Asylpaket I wurde den Landesregierungen zum Oktober 2015 ermöglicht, die gesetzliche Krankenversicherung mit der Gesundheitsversorgung von Asylsuchenden zu beauftragen. Nach Reiter und Töller (2019, S. 203) hatte dies zwei Änderungen zur Folge: Erstens können die Erbringer der Leistungen (Ärzte, Psychologen etc.) diese unmittelbar mit der Krankenkasse abrechnen und erhalten hierfür eine Verwaltungspauschale, welche der Kommune in Rechnung gestellt werden kann. Zweitens müssen Asylsuchende durch den Erhalt einer elektronischen Gesundheitskarte nicht wie zuvor in jedem Einzelfall eine Berechtigung beim Sozialamt erwerben. Die Gesundheitskarte bietet somit weniger bürokratischen Aufwand und erleichtert den Geflüchteten eine schnellere, fachlich bessere und menschenwürdigere Gesundheitsversorgung. All dies sind Gründe, weshalb die Einführung einer Gesundheitskarte als permissive Maßnahme verstanden werden kann.

Ob ein Land die Gesundheitskarte jedoch einführt, bleibt ihm selbst überlassen (Wächter-Raquet 2016, S. 4). Die dadurch sichtbare Varianz kann - einem Vorschlag der Bertelsmann Stiftung folgend - anhand mehrerer Kriterien genauer zu 
vermessen werden ${ }^{4}$ : (1) Haltung zur Einführung der Gesundheitskarte (Ablehnung, Zustimmung), (2) Rahmenbedingungen (Gespräche mit Krankenkassen), (3) Kostenübernahme durch das Bundesland und (4) landesweite Regelungen (siehe dazu auch: Günther et al. (2019)). Mit Blick auf das erste Kriterium besteht die Varianz darin, dass einige Bundesländer wie Bayern oder Sachsen die Einführung ablehnten, während andere wie Berlin, Bremen, Hamburg, Niedersachsen, Nordrhein-Westfalen, Schleswig-Holstein und Thüringen zustimmten. Daneben gibt es in der Umsetzung selbst Unterschiede: Brandenburg und Rheinland-Pfalz haben die Gesundheitskarte verzögert umgesetzt, während Baden-Württemberg, Hessen, Mecklenburg-Vorpommern, das Saarland und Sachsen-Anhalt auf die Einführung verzichteten, nachdem Gespräche mit den Krankenkassen scheiterten. Bayern und Sachsen hingegen lehnten im Vorfeld die Einführung ab, weshalb es erst gar nicht zu Gesprächen kam. Alle anderen Bundesländer kamen zu einem Abschluss mit den Krankenkassen. Mit Blick auf die Kosten unterscheiden sich die Länder darin, ob die Kosten vollständig durch das Land übernommen werden (Brandenburg, Schleswig-Holstein und Thüringen), oder ob in einem „Teilmodell“ die Kosten auf die Kommunen übertragen werden und diese dann freiwillig entscheiden können, ob eine Gesundheitskarte für Geflüchtete eingeführt wird (Niedersachsen, Nordrhein-Westfalen, RheinlandPfalz). ${ }^{5}$ Zudem kann untersucht werden, ob eine landesweite verbindliche Regelung vorliegt oder die einzelnen Kommunen entscheiden bzw. ob sich in diesem Fall viele Kommunen daran beteiligen. Landesweite Regelungen sind in Brandenburg, Berlin, Bremen, Hamburg und Schleswig-Holstein zu finden. Niedersachsen und RheinlandPfalz haben keine landesweiten Regelungen, während diese in Nordrhein-Westfalen teilweise festzustellen sind.

\subsection{Einrichtung von Ankerzentren}

Im „Masterplan Migration“ von Bundesinnenminister Horst Seehofer wurde die Einrichtung von sogenannten Ankerzentren festgelegt (Bundesministerium des Innern, für Bau und Heimat (BMIBH) 2018, S. 14ff.). Ziel der Ankerzentren ist es, die Kompetenzen von Bund, Ländern und Kommunen in einem Zentrum zusammenzuführen. Es gilt für die Asylsuchenden eine Residenzpflicht und ein Arbeitsverbot, darüber hinaus sollen Sachleistungen konsequent Vorrang vor Geldleistungen haben. Zwischen 1000 und 1500 Asylsuchende sind für bis zu 18 Monate (Familien: 6 Monate) in Ankerzentren untergebracht; sie werden erst dann auf die Kommunen verteilt, wenn ihr Antrag positiv beschieden worden ist (BMIBH 2018, S. $14 \mathrm{ff}$.). Zudem werden keine Sprach- oder Integrationskurse angeboten und der Besuch von Beratern der Wohlfahrtsverbände ist nicht gestattet bzw. genehmigungspflichtig (Reiter und Töller 2019, S. 210). Der Kern des Konzepts ist also eine Kasernierung der Asylsuchenden und im Ablehnungsfall eine effiziente Rückführung (Schammann

\footnotetext{
${ }^{4}$ Der ursprüngliche Datensatz der Bertelsmann Stiftung, welcher im Jahr 2016 im Februar veröffentlicht und später im Mai ergänzt wurde, ist jedoch sehr lückenhaft und somit wenig aussagekräftig. Daher haben wir diesen durch eigene Recherche ergänzt.

5 In den Stadtstaaten Berlin, Bremen und Hamburg stellt sich die Frage der Kostenübernahme nicht. In Bremen kam ein direkter Abschluss mit der Stadt Bremen sowie dem Magistrat Bremerhaven zustande.
} 
2018). Daher wird die Einrichtung von Ankerzentren als Ausdruck einer restriktiven Migrationspolitik angesehen.

Die Bundesländer können vom Bund allerdings nicht zur Errichtung von Ankerzentren verpflichtet werden, weil die Länder für die Unterbringung und die Rückführung von Asylbewerbern zuständig sind. Der Bund spricht nur Empfehlungen aus. Diese Freiheit nutzen die Länder, wobei Unterschiede erst im Detail sichtbar werden. Reiter und Töller (2019, S. 210 ff.) differenzieren in ihrer Untersuchung drei Ländercluster: Die erste Gruppe habe Ankerzentren eingerichtet, die zweite Gruppe der Bundesländer habe mitgeteilt, dass sie bereits über Einrichtungen verfügen, die die Funktionen der Ankerzentren erfüllen würden, und die dritte Gruppe lehne die Einrichtung von Ankerzentren ausdrücklich ab. Weil mittlerweile alle 16 Bundesländer angeben, Einrichtungen zu haben, die die Funktion von Ankereinrichtungen erfüllen, differenzieren wir die Länder in diesem Aufsatz weiter aus. Die erste Gruppe vereinigt die Länder, die eine Vereinbarung mit dem Bund über die Einrichtung von Ankerzentren abgeschlossen haben. Hierzu gehören Bayern und Sachsen, die bereits am 1. August 2018 die ersten Ankerzentren öffneten, sowie das Saarland, das zwei Monate später nachnachzog. Ebenfalls in diese Gruppe - jedoch weniger eindeutig - fallen Mecklenburg-Vorpommern und Schleswig-Holstein. Beide Länder haben eine Vereinbarung zur Einrichtung eines Ankerzentrums abgeschlossen, verzichten jedoch auf die Nutzung des Namens (NDR 2019). In Schleswig-Holstein wird etwa stattdessen der Begriff des „Kompetenzzentrums“ genutzt, das jedoch die Aufgabe eines Ankerzentrums erfüllt (Kieler Nachrichten 2019).

Eine zweite Gruppe von Bundesländern hat zwar keine Vereinbarung mit dem Bundesinnenminister abgeschlossen, verweist aber ausdrücklich darauf, dass sie bereits über Erstaufnahmeeinrichtungen verfügen, die das Konzept der „Ankerzentren" funktionsgleich umsetzen - etwa in Niedersachsen und Hessen (Tagesschau 2019) oder in Hamburg (Zeit Online 2019). Auch Sachsen-Anhalt gibt an, dass mit der zentralen Anlaufstelle für Asylbewerber in Halberstadt fast alle Funktionen eines Ankerzentrums umgesetzt würden. Ein Konzept sehe zudem den Ausbau eines zweiten Standorts in Stendal vor.

Schließlich gibt es eine Gruppe von Bundesländern, die das Konzept der „Ankerzentren" nahezu vollständig ablehnen. Die Landesregierung von Rheinland-Pfalz sieht das Modell einer integrierten Erstaufnahme mit allen beteiligten Stellen unter einem Dach bereits erfolgreich umgesetzt. Weiterführende Maßnahmen, die Teil des Konzepts der „Ankerzentren“ sind, lehnt die Landesregierung allerdings ab. Auch Bremen, Berlin, Thüringen, Nordrhein-Westfalen und Baden-Württemberg hatten schon früh abgelehnt, „Ankerzentren“ einzurichten und sich offen gegen das Konzept gestellt. Demzufolge wird neben der Einrichtung von „Ankerzentren“ zudem der Sonderfall unterschieden, dass es wenige Bundesländer gibt, die zwar eine Vereinbarung zur Umsetzung des Konzepts unterzeichnet haben, aber die offizielle Bezeichnung „Ankerzentrum“ ablehnen. Zudem wird der Verweis auf ein funktionales Äquivalent durch die Landesregierung eines Bundeslandes ebenfalls als Ausdruck einer eher restriktiven Migrationspolitik gewertet. Die Ablehnung einer solchen Einrichtung wird hingegen als permissive Maßnahme gewertet. 


\subsection{Abschiebepraxis nach Afghanistan}

Als fünfte Dimension der Vermessung der Migrationspolitik der Länder ziehen wir die Abschiebepraxis der Bundesländer nach Afghanistan im Jahr 2017 heran, da Afghanistan im Bereich von Abschiebungen als paradigmatischer Fall anzusehen ist (Hörisch 2018). Zentrale Frage hierbei ist die Bewertung des Landes als ,sicheres Herkunftsland ", die aufgrund der volatilen Sicherheitslage umstritten ist. Grundsätzlich stellt die Durchführung von Abschiebungen eine Kompetenz der Bundesländer dar. Die Zuständigkeit für den Vollzug der Abschiebung, d.h. für die Beförderung bis zur Grenzbehörde liegt, nach § 71 Absatz 5 des Aufenthaltsgesetzes (AufenthG), bei den Polizeien der Länder. Allein die Rückführung von einer Grenzbehörde, beispielsweise einem Flughafen, fällt in die Zuständigkeit der Bundespolizei (§ 71, Abs. 3, Nr. 1d). ${ }^{6}$

Die Möglichkeit von Abschiebungen nach Afghanistan hat sich über die Zeit mehrmals verändert. Zunächst forderte das Bundesinnenministerium auf Grundlage eines Rückführungsabkommens (Oktober 2016) eine konsequente Abschiebung nach Afghanistan (Spiegel Online 2017), nach einer Verschlechterung der Sicherheitslage wurden die Abschiebungen im Sommer 2017 vorübergehend vom Bund auf Gefährder, Straftäter und sogenannte Identitätstäuscher beschränkt - die Innenministerkonferenz folgte dieser Einschätzung. Im Juni 2018 hob die Bundesregierung ihre Einschränkungen wieder auf, nachdem das Auswärtige Amt den aktuellen Lagebericht zur Sicherheitslage in Afghanistan veröffentlicht hatte (Tagesschau 2018). Diese Einschätzung wird von einem Großteil der Bundesländer nicht geteilt, auch wenn die Innenministerkonferenz (Juni 2018) ebenfalls die Abschiebebeschränkung auf die drei genannten Gruppen (Straftäter, Gefährder, Identitätsverweigerer) aufgehoben hat. Varianz ist die Folge. Einige Länder schieben weiterhin nur Gefährder, Straftäter und Identitätsverweigerer ab (z. B. Rheinland-Pfalz) ${ }^{7}$, Bayern und Sachsen beschränken ihre Abschiebungen jedoch nicht auf diese Gruppe, wobei Sachsen Frauen und Kinder ausnimmt. In Einzelfällen schieben auch Brandenburg, Mecklenburg-Vorpommern und Sachsen-Anhalt Migranten nach Afghanistan ab, die nicht zu der Gruppe der Straftäter, Gefährder und Identitätsverweigerer gehören (Süddeutsche Zeitung 2019). Die Landesregierung in Mecklenburg-Vorpommern will künftig alleinstehende Männer nach Afghanistan rückführen, die sich weder in einer Ausbildung befinden noch eine Arbeit aufgenommen haben (Tagesschau 2018). Lediglich

\footnotetext{
${ }^{6}$ Eine alternative Möglichkeit wäre, entlang des Vorschlags von Reiter und Töller auch die Rückführungsquoten mit in den Blick zu nehmen. Da zu diesen jedoch auch freiwillige Ausreisen gezählt werden, ist eine klare Einordnung als ,restriktive“ Maßnahme weniger eindeutig als im Falle der Abschiebepraxis nach Afghanistan, die als ,paradigmatischer Fall“ auch stark politisiert war. Daher liegt für uns eine landespolitische Beeinflussung im Fall der Abschiebung nach Afghanistan näher. Empirisch zeigen sich ohnehin in nur 3 von 16 Fällen größere Abweichungen zwischen der allgemeinen Abschiebepraxis und dem Fall Afghanistan: Bayern ist nach unserer Messung sehr restriktiv, landet in der Abschiebepraxis aber im Mittelfeld. Der umgekehrte Befund gilt für das Saarland und Schleswig-Holstein. Das Saarland schiebt sehr konsequent ab, liegt im Fall Afghanistan jedoch im Mittelfeld (nur Straftäter, Identitätsverweigerer und Gefährder werde abgeschoben); Schleswig-Holstein hingegen liegt im Mittelfeld bei der Abschiebepraxis, verordnete sich jedoch im Februar 2017 aus humanitären Gründen einen Abschiebestopp (ausgenommen: Straftäter).

${ }^{7}$ Siehe LT-Drs. 17/2506 (Rheinland-Pfalz).
} 
Schleswig-Holstein ordnete im Februar 2017 in einer Koalition aus SPD, Grünen und SSW einen formalen Abschiebestopp nach § 60a Aufenthaltsgesetz an, wonach ein Landesinnenministerium ,,aus völkerrechtlichen oder humanitären Gründen [...] anordnen [kann], dass die Abschiebung von Ausländern aus bestimmten Staaten oder von in sonstiger Weise bestimmten Ausländergruppen allgemein oder in bestimmte Staaten für längstens drei Monate ausgesetzt wird“. Straftäter sind hiervon jedoch auch in Schleswig-Holstein ausgenommen. In anderen Bundesländern, wie Rheinland-Pfalz oder Niedersachsen, ergibt sich der Abschiebestopp de facto. Diese Länder führen Einzelfallprüfungen durch, in denen dann die Sicherheitslage eine Abschiebung jeweils verhindert. Aufgrund einer solchen Einzelfallprüfung schieben diese Länder dann ebenfalls nur Straftäter, Gefährder und Identitätsverweigerer ab (Reiter und Töller 2019, S. 208). Einen weiteren Sonderfall stellt der Stadtstaat Bremen dar. Demnach gilt in Bremen eine Weisung des Innensenators, dass die Bremer Ausländerbehörden nur Straftäter oder Personen, die eine falsche Identität vorgetäuscht haben, nach Afghanistan abschieben dürfen. Darüber hinaus lehnt Bremen eine Beteiligung an Sammelabschiebungen nach Afghanistan als einziges Bundesland ausdrücklich ab (Röhlung und Rabba 2018).

In der Summe ergeben sich zwei Gruppen - Länder die generell abschieben und Länder, die nur Straftäter, Gefährder und Identitätsverweigerer abschieben. Die konkrete Praxis der Länder lässt sich jedoch noch weiter ausdifferenzieren, sodass im Ergebnis vier Ländercluster identifizieren lassen:

1. Keine Sammelabschiebungen bzw. Formaler Abschiebestopp: Bremen, Schleswig-Holstein;

2. Abschiebung von Straftätern, Gefährdern und Identitätsverweigerern: BadenWürttemberg, Berlin, Hamburg, Hessen, Niedersachsen, Nordrhein-Westfalen, Rheinland-Pfalz, Saarland, Thüringen;

3. Nur in Einzelfällen Abschiebung von Personen, die nicht zur Gruppe von Straftätern, Gefährdern und Identitätsverweigerern zählen: Brandenburg, MecklenburgVorpommern und Sachsen-Anhalt;

4. Generelle Abschiebung: Bayern, Sachsen.

\subsection{Bundesratsabstimmung zu sicheren Herkunftsländern ,Maghreb-Staaten“}

Als letzte Dimension der Migrationspolitik der Bundesländer wird das Abstimmungsverhalten der Bundesländer im Bundesrat zum Gesetzesentwurf zur Ausweitung der sicheren Herkunftsländer um die sogenannten Maghreb-Staaten (Algerien, Tunesien und Marokko) im März 2017 herangezogen. Diese Bundesratsabstimmung eignet sich deshalb besonders gut, um die Migrationspolitik der Landesregierungen $\mathrm{zu}$ vermessen, da das Gesetz und das Abstimmungsverhalten öffentlich kontrovers diskutiert wurden. Für die Landesregierungen stellte die Entscheidung also eine Gelegenheit dar, die Ausrichtung ihrer Migrationspolitik öffentlich darzustellen was sich auch darin zeigte, dass Baden-Württemberg große Schwierigkeiten hatte, mit dem erreichten „Package Deal“" in der Öffentlichkeit durchzudringen (vgl. Abschn. 6). Das Gesetz war vor allem wegen der Menschenrechtslage in den drei Maghreb-Ländern umstritten. Sichere Herkunftsstaaten sind nach § 29 des Asyl- 
verfahrensgesetzes solche Länder, in denen weder politische Verfolgung noch unmenschliche oder erniedrigende Bestrafung oder Behandlung stattfindet. Asylanträge von Menschen aus diesen Staaten werden in der Regel abgelehnt, solange die Betroffenen nicht glaubhaft nachweisen können, dass sie doch verfolgt werden.

Das Gesetz zur Ausweitung der sicheren Herkunftsländer um die Maghreb-Staaten wurde im Mai 2016 im Bundestag verabschiedet, eine Abstimmung im Bundesrat erfolgte jedoch mangels absehbarer Mehrheit nicht. Auf Antrag Bayerns wurde das Gesetz Anfang 2017 erneut auf die Tagesordnung des Bundesrates gesetzt, fand jedoch im März 2017 keine Mehrheit, da sich bis auf Baden-Württemberg ${ }^{8}$ alle Landesregierungen mit Regierungsbeteiligung von Grünen und Linken enthielten. Berlin stimmte gegen den Entwurf, da sich der Berliner SPD-Landesvorstand mit großer Mehrheit gegen die Einstufung der Maghreb-Staaten Marokko, Tunesien und Algerien als sichere Herkunftsstaaten ausgesprochen hatte (Tagesspiegel 2018). Auch ein erneuter Anlauf in der neuen Legislaturperiode (im Januar 2019) fand angesichts unveränderter Verhältnisse im Bundesrat bisher keine Mehrheit in der Länderkammer (FAZ 2019). Daher ergeben sich zwei Ländergruppen: Baden-Württemberg, Bayern, Mecklenburg-Vorpommern, Saarland und Sachsen haben dem Gesetz zugestimmt, wohingegen sich Brandenburg, Bremen, Hamburg, Hessen, Niedersachsen, Nordrhein-Westfalen, Rheinland-Pfalz, Sachsen-Anhalt, Schleswig-Holstein und Thüringen enthalten haben. $\mathrm{Zu}$ dieser Gruppe zählt auch Berlin, da eine Ablehnung im Bundesrat faktisch einer Enthaltung gleichkommt.

\subsection{Muster der Migrationspolitik: ein Zwischenfazit}

Betrachtet man die Migrationspolitik der 16 deutschen Länder in den sechs ausgewählten Dimensionen, so zeigen sich bereits auf den ersten Blick bestimmte Muster (vgl. Tab. 1). Auf Seiten der besonders restriktiven Länder stechen Bayern und Sachsen heraus, die in den meisten der Dimensionen sehr ähnlich restriktive Entscheidungen getroffen haben. Auf der anderen Seite stehen Länder wie Bremen, Berlin oder auch Rheinland-Pfalz, die in ihren migrationspolitischen Entscheidungen eher permissiv agiert haben. Schließlich gibt es aber auch eine ganze Reihe von Fällen mit spezifischen Profilen, bei denen sich die Entscheidungen in bestimmten Dimensionen spezifisch erklären lassen - wie etwa die Zustimmung Baden-Württembergs zu den sicheren Herkunftsländern bei sonst eher permissivem Profil in der Migrationspolitik. Die hier dargestellten Dimensionen werden in der späteren Analyse zu einem Outcome zusammengeführt, die Kalibrierung erfolgt in Abschn. 4.

\footnotetext{
8 Obwohl Baden-Württemberg mit Winfried Kretschmann einen grünen Ministerpräsidenten hat, stimmte es als einziges Bundesland mit grüner Regierungsbeteiligung dem Gesetzesentwurf zu. Ministerpräsident Winfried Kretschmann wurde für das Abweichen von der bundesweiten Parteilinie in den eigenen Reihen schwer kritisiert (Stuttgarter Zeitung 2018). Kretschmann begründete seine Zustimmung zu dem Gesetzesentwurf damit, dass die Bundesregierung zugesagt habe, gefährdete Gruppen so zu behandeln wie bisher, sodass der Menschenrechtsfrage ein Stück weit Genüge getan sei.
} 


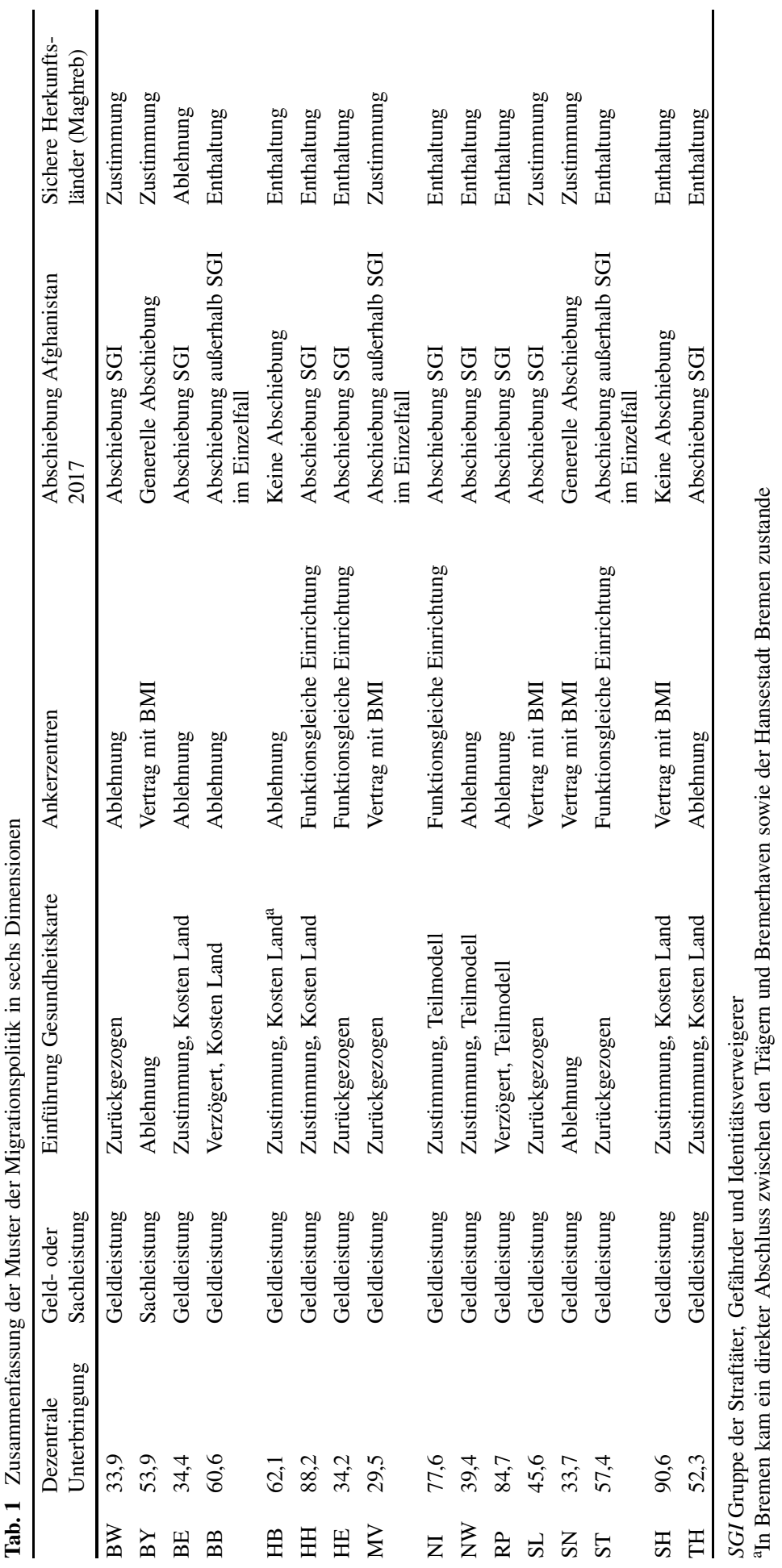




\section{Theoretische Vorüberlegungen}

Neben der mehrdimensionalen Vermessung der Migrationspolitik in den deutschen Ländern zielen wir in diesem Aufsatz auch darauf ab, mögliche Bedingungskonstellationen herauszuarbeiten, welche die Varianz zwischen den Ländern erklären können. Theoretisch greifen wir dabei auf etablierte Theorieansätze der Policy-Forschung zurück (Wenzelburger und Zohlnhöfer 2015), ergänzen diese jedoch durch politikfeldspezifische Erklärungen. Zudem schlagen wir für die Untersuchung des parteipolitischen Einflusses auf die Migrationspolitik eine Messung der parteipolitischen Position der Regierung unter Rückgriff auf Parteiprogramme zurück - eine Neuerung im Vergleich zum bisherigen Forschungsstand. Insgesamt vereinigt unser Theoriemodell drei zentrale Bedingungen, von denen - auch auf Grundlage des Forschungsstands - erwartet wird, dass sie in ihrer Kombination zur Erklärung der unterschiedlichen Outcomes in den Bundesländern beitragen.

Bestehende Studien zur Migrationspolitik haben häufig einen Einfluss der parteipolitischen Ausrichtung der Regierung festgestellt (Günther et al. 2019; Hörisch 2018). Daher gehen auch wir von einem Erklärungsbeitrag der Parteiendifferenztheorie (Schmidt 1993) aus. Allerdings scheint uns eine schlichte Einordnung der Regierungsparteien in den Ländern auf einer Links-rechts-Achse zu kurz zu greifen, weshalb wir die häufige Praxis der Untersuchung von Kabinettssitzanteilen von Parteifamilien hier nicht nutzen und stattdessen die programmatischen Positionen der Landesverbände zu Fragen von Migration und Asyl in unsere Analyse aufnehmen (dazu: Wenzelburger 2015). Grundlage unserer Einschätzung sind die Ergebnisse einer aktuellen Auswertung der Parteipositionen durch Bräuninger und Kollegen (Bräuninger et al. 2020), die mittels eines Wordscore-Verfahrens die Landesparteien mit Blick auf deren migrationspolitische Programmatik verortet haben, wobei hohe Werte eine autoritäre Position und niedrige Werte eine liberale Position anzeigen. ${ }^{9}$ Eine nach Landesverbänden aufgegliederte Auswertung der migrationspolitischen Position ist schon allein deshalb sinnvoll, da die Parteien in den Ländern durchaus unterschiedliche Positionen einnehmen (vgl. Abb. 1). So positionieren sich der baden-württembergischen Landesverbands der Grünen mit einem Wert 7,4 und die saarländischen Grünen $(8,2)$ deutlich stärker autoritär als die der Grünen in Bremen $(3,0)$ oder in Sachsen-Anhalt $(0,3)$. Ähnlich Varianz zeigt sich bei der FDP, deren migrationspolitische Position in einigen Ländern autoritärer ausfällt als die der CDU (etwa in Sachsen-Anhalt oder im Saarland), nicht aber in Bayern. Daher wird bei der Vermessung der ideologischen Positionierung einer Landesregierung die Positionen der an der Regierung beteiligten Landesparteien berücksichtigt (s. unten). Unsere erste Hypothese (H1) lautet daher, dass eine autoritäre migrationspolitische Position der Regierung mindestens eine INUS-Bedingung ${ }^{10}$ für eine restriktive Migra-

\footnotetext{
9 Wir danken Marc Debus und dem Mannheimer Team für die Möglichkeit, diese Daten zu nutzen.

10 Unter einer INUS-Bedingung versteht man eine Bedingung, die notwendiger Teil einer kombinierten Bedingung für ein Outcome ist, zu der es jedeoch weitere Alternativen gibt (Wagemann 2015, S. 433). Da wir in dieser Studie keine starken theoretischen Vorannahmen für bestimmte Bedingungskonstellationen oder die Notwendigkeit einzelner Bedingungen oder Kombinationen dieser haben, scheint uns eine offene Formulierung im Sinne von INUS-Bedingungen angemessen.
} 

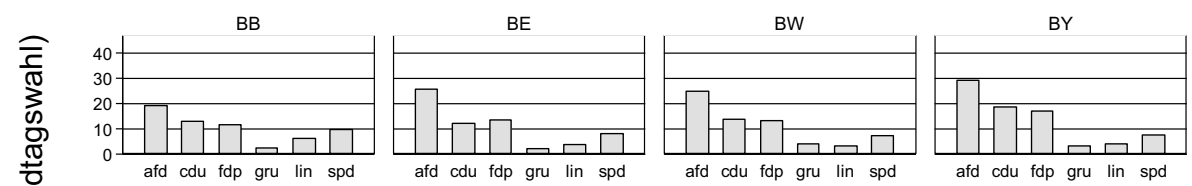

HB
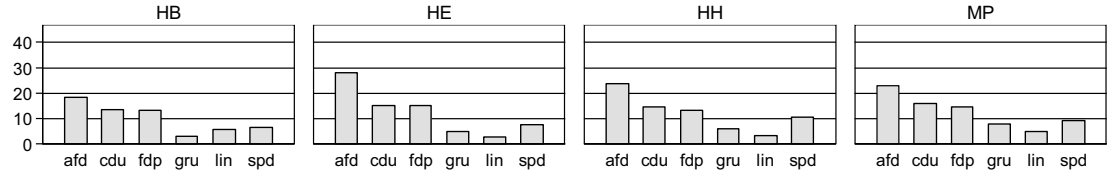

$\mathrm{N}$
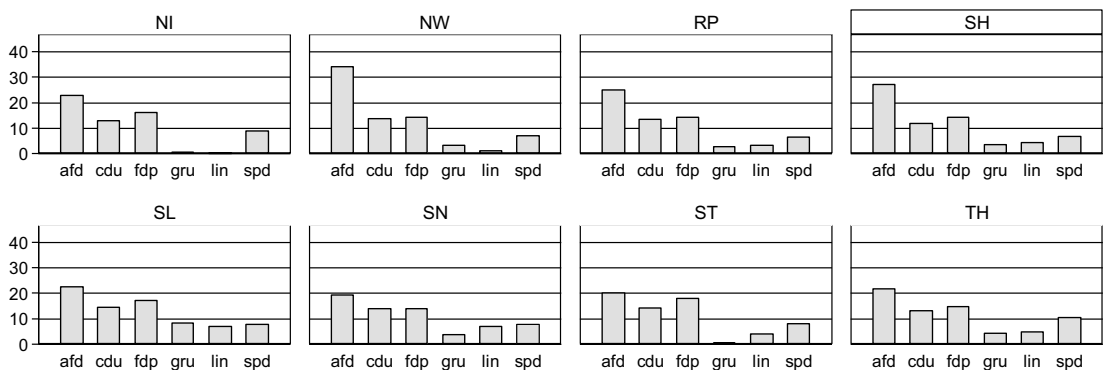

$\mathrm{TH}$

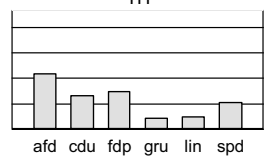

Abb. 1 Migrationspolitische Position der Landesverbände ( $B B$ Brandenburg, $B E$ Berlin, $B W$ BadenWürttemberg, $B Y$ Bayern, $H B$ Bremen, $H E$ Hessen, $H H$ Hamburg, $M P$ Mecklenburg-Vorpommern, NI Niedersachsen, $N W$ Nordrhein-Westfalen, $R P$ Rheinland-Pfalz, $S H$ Schleswig-Holstein, SL Saarland, SN Sachsen, ST Sachsen-Anhalt, TH Thüringen. In Bayern wird die CSU hier als CDU benannt. Das Kürzel lin steht für die Linkspartei. Es wurden nur die im Bundestag vertretenen Parteien ausgewertet. Hohe Werte auf der y-Achse deuten eine autoritäre migrationspolitische Position an). (Nach Bräuninger et al. 2020)

tionspolitik darstellt (und spiegelbildlich ${ }^{11}$ für eine liberale Position und permissive Politik).

Vergleichende Policy-Analysen zu den Bundesländern haben in den vergangenen Jahren häufig gezeigt, dass die wirtschaftlichen und finanzpolitischen Rahmenbedingungen die Politik in den deutschen Ländern stark beeinflussen (z. B. Hildebrandt und Wolf 2016). Angesichts der budgetären Kosten, welche die Migrationspolitik gerade in den letzten Jahren für die Landeshaushalte versursacht hat, gehen wir davon aus, dass die ökonomischen Rahmenbedingungen für migrationspolitische Entscheidungen relevante Kontextfaktoren sind. Wir folgen dabei der Argumentation von Hörisch (2018, S. 789) wie auch von Günther et al. (2019, S. 380) und gehen davon aus, dass eine gute sozio-ökonomische Ausgangslage zu einer Bedingungskonstellation beiträgt, die eine permissivere Migrationspolitik ermöglicht. Eine geringere Verschuldung ermöglicht es den Bundesländern, eine kostenintensivere liberale Integrationspolitik zu betreiben. Eine starke Wirtschaftskraft dürfte gerade vor dem Hintergrund des Fachkräftemangels eine positivere Sicht auf die Notwendigkeit von Zuwanderung zur Folge haben und eine geringe Arbeitslosenquote die Akzeptanz von Migration in der Bevölkerung erhöhen (dazu auch: Hörisch 2018, S. 788-789).

11 Auch wenn asymmetrische Mengenbeziehungen gerade durch QCA gut herausgearbeitet werden können, haben wir hierfür keine starken theoretischen Hinweise und vermuten daher Symmetrie. Dies gilt ebenfalls für die weiteren Hypothesen. 
Folglich erwarten wir mit $\mathrm{H} 2$, dass schlechtere sozio-ökonomische Rahmenbedingungen mindestens eine INUS-Bedingung für eine restriktive Migrationspolitik darstellen (und spiegelbildlich für bessere Rahmenbedingungen und permissive Politik).

Unsere dritte Bedingung, deren Relation zu einer restriktiven bzw. permissiven Migrationspolitik untersuchen wollen, greift auf die Einstellungen der Landesbevölkerung zurück. Ausgehend von der Annahme, dass Regierungen nicht nur PolicySeeking betreiben, sondern auch wiederwahlorientiert sind (Müller und Strøm 1999), sollten sich die Präferenzen der Wählerinnen und Wähler in den Bundesländern auf die Migrationspolitik auswirken. Diese Annahme lässt sich zudem mit Ergebnissen zur Kontakthypothese (Allport 1954; Pettigrew und Tropp 2006) zusammenführen, die nahelegt, dass eine vergleichswiese einfache Maßnahme, nämlich Kontakt, die Einstellung gegenüber Unbekannten - in diesem Falle den Geflüchteten - positiv beeinflusst (zur Flüchtlingskrise: Landmann et al. 2018; Becker et al. 2018; Kotzur 2018). Für den Ausländeranteil als Maß für Kontakt hat Stürmer (2008) zwar gezeigt, dass ein entsprechender Zusammenhang mit der Akzeptanz permissiver Migrationspolitik durch die Bürger vorliegt; Für migrationspolitische Maßnahmen der Landesregierungen ist die empirische Evidenz jedoch gemischt (unterstützend: Hörisch 2018; ablehnend: Günther et al. 2019). Wir greifen daher auf die Einstellungen der Bevölkerung zurück (s. unten), um das „Vote-Seeking“ der Landesregierungen direkter zu messen. Unsere dritte Hypothese lässt sich daher wie folgt formulieren (H3): Eine negative Einstellung der Bevölkerung eines Bundeslandes ist mindestens eine INUS-Bedingung für eine restriktive Migrationspolitik (und spiegelbildlich für positive Einstellungen und permissive Politik).

\section{Methodisches Vorgehen und Kalibrierung der Daten}

Die unterschiedliche Migrationspolitik der 16 deutschen Bundesländer wird in der Folge mittels einer fuzzy-set Qualitative Comparative Analysis (QCA) untersucht. Die von Charles C. Ragin (1987) entwickelte Methode soll nicht nur die mangelnde Generalisierbarkeit einer fallorientierten Forschung mit kleiner Fallzahl und den Komplexitätsmangel einer statistischen, variablenorientierten Herangehensweise mit großer Fallzahl ausgleichen, sondern auch durch ein komplexeres Kausalverständnis der Komplexität sozialer Phänomene Rechnung tragen (Schneider und Wagemann 2012). Für die Policy-Analyse ist gerade die Berücksichtigung von Äquifinalität eine Stärke der Methode, da politische Prozesse häufig kontextabhängig unterschiedlich verlaufen und nicht ein einziges Erklärungsmodell für alle Fälle zutrifft. Aufgrund ihrer Ausrichtung auf eine mittelgroße Fallzahl und ihr komplexes Kausalitätsverständnis ist die QCA gut auf die Untersuchung der Migrationspolitik der Bundesländer anwendbar. Wir konzedieren, dass angesichts der begrenzten Zahl von drei Bedingungen in unserer Studie kein Anspruch auf vollständige kausale Erklärung des Outcomes erhoben werden kann. Aus unserer Sicht eignet sich unser Vorgehen jedoch, um Muster in den Daten freizulegen und unter Berücksichtigung qualitativer Evidenz, theoretischer Vorüberlegungen und dem Forschungsstand durchaus vorsichtige Schlüsse im Sinne einer Erklärung der Varianz zu ziehen. 
Im Kern geht es der QCA darum, eine Mengenbeziehung zwischen einem Outcome (hier: permissive oder restriktive Migrationspolitik) und theoretisch hergeleiteten Bedingungen herzustellen, von denen ein kausaler Zusammenhang mit dem Outcome erwartet wird. Ziel der folgenden Analyse ist es also zu prüfen, inwiefern Parteiideologie, sozio-ökonomischer Kontext und die Einstellungen der Bevölkerung zu Geflüchteten allein oder in ihrer Kombination hinreichend oder notwendig für die Restriktivität bzw. Permissivität der Migrationspolitik der Bundesländer sind. Durch die Identifikation unterschiedlicher Pfade zu zwei Outcomes kann die QCA auch mit Asymmetrie umgehen, d.h. das Vorliegen einer Bedingung für den Outcome Restrikitivität muss nicht automatisch bedeuten, dass die Abwesenheit der Bedingung auch zum Outcome Permissivität führt. Der Logik der QCA folgend präsentieren wir die Ergebnisse daher sowohl für den Outcome Restriktivität als auch für den Outcome Permissivität und führen jeweils eine Analyse der notwendigen wie auch der hinreichenden Bedingungen durch.

Für die fsQCA müssen in einem ersten Schritt das Outcome wie auch die Bedingungen auf den Wertebereich von 0-1 kalibriert werden. Dies erfordert zunächst eine Entscheidung darüber, ob eine Beobachtung (hier ein Land) innerhalb oder auBerhalb einer Menge mit Blick auf das Outcome oder die Bedingung liegt, und im zweiten Schritt die Feststellung des Ausmaßes der Mengenmitgliedschaft (voll oder teilweise). Angesichts des mehrdimensionalen Messkonzepts der Migrationspolitik umfasst die Kalibrierung des Outcomes die Betrachtung aller Dimensionen und deren Reduktion auf einen fuzzy-Wert für jedes Bundesland. Hierzu wurden - auch um die Robustheit der Ergebnisse für die Kalibrierungen nachzuweisen - zwei unterschiedliche Vorgehensweisen gewählt. Im ersten Fall, der auch im Folgenden berichtet wird, haben wir in einem ersten Schritt die Rohdaten für die sechs Dimensionen auf eine Skala von 0-1 standardisiert (zum Vorgehen, siehe Appendix). Wie aus Tab. 2 ersichtlich ist sind die meisten der festgestellten Unterschiede qualitativer Natur und beschreiben eine ordinale Skala - etwa mit Blick auf das Abstimmungsverhalten im Bundesrat, die Abschiebung nach Afghanistan oder die Ankerzentren. Für die dezentrale Unterbringung liegen hingegen ebenso metrische Werte vor wie für die Dimension „Gesundheitskarte“, deren Umsetzungsgrad durch die Bertelsmann-Stiftung auf einer Skala von 0-12 verortet wurde. Schließlich unterscheidet sich die Art der Existenzsicherung für Geflüchtete nur in zwei Ausprägungen ob Geld- oder Sachleistungen gezahlt werden. Einmal auf eine 0-1-Skala gebracht wurden die Dimensionen über das arithmetische Mittel zusammengeführt und im nächsten Schritt direkt kalibriert. Hierbei wurden die Schwellenwerte nach visueller Inspektion der Verteilung der Variablen gesetzt (s. Appendix), wobei sich in der Kalibrierung vier Wertestufen als sinnvoll erwiesen.

Im zweiten Fall (alle Darstellungen, s. Appendix) wurde anhand der qualitativen Einschätzungen jeweils für jede einzelne Dimension (bis auf die Dezentralisierung) eine vierstufige Mengenmitgliedschaft in der Menge der restriktiven Maßnahmen festgestellt (,fully in“, ,more in than out“, ,more out than in“" und ,fully out"). Für die Dezentralisierung wurde auf Basis der metrischen Daten ebenfalls eine Mengenmitgliedschaft durch Betrachtung der Verteilung der Daten festgestellt. Die Zusammenführung erfolgte sodann erneut qualitativ durch Gegenüberstellung der Mitgliedschaften in der repressiven Menge für die einzelnen Dimensionen (vgl. Ap- 
Tab. 2 Kalibrierung Outcome: Restriktive Migrationspolitik

\begin{tabular}{llllllllll}
\hline & $\begin{array}{l}\text { Gesund- } \\
\text { heits- } \\
\text { karte }\end{array}$ & $\begin{array}{l}\text { Anker- } \\
\text { zentrum }\end{array}$ & $\begin{array}{l}\text { Dezentrale } \\
\text { Unter- } \\
\text { bringung }\end{array}$ & $\begin{array}{l}\text { Ab- } \\
\text { schie- } \\
\text { bung }\end{array}$ & $\begin{array}{l}\text { Geld- } \\
\text { leis- } \\
\text { tungen }\end{array}$ & $\begin{array}{l}\text { Abstimmung Mittel- } \\
\text { Bundes- } \\
\text { rat }\end{array}$ & $\begin{array}{l}\text { Fs- } \\
\text { wert }\end{array}$ & $\begin{array}{l}\text { Fs-score } \\
\text { score } \\
\text { (alterna- } \\
\text { tiv) }\end{array}$ \\
\hline BB & 0,92 & 1,00 & 0,38 & 0,25 & 1,00 & 0,67 & 0,70 & 0,33 & 0,33 \\
BE & 1,00 & 1,00 & 0,67 & 0,50 & 1,00 & 1,00 & 0,86 & 0 & 0 \\
BW & 0,00 & 1,00 & 0,37 & 0,50 & 1,00 & 0,00 & 0,48 & 0,66 & 0,66 \\
BY & 0,00 & 0,00 & 0,59 & 0,00 & 0,00 & 0,00 & 0,10 & 1 & 1 \\
HB & 0,83 & 1,00 & 0,69 & 1,00 & 1,00 & 0,67 & 0,86 & 0 & 0 \\
HE & 0,00 & 0,75 & 0,38 & 0,50 & 1,00 & 0,67 & 0,55 & 0,66 & 0,33 \\
HH & 1,00 & 0,75 & 0,97 & 0,50 & 1,00 & 0,67 & 0,82 & 0,33 & 0,33 \\
MV & 0,00 & 0,50 & 0,33 & 0,25 & 1,00 & 0,00 & 0,35 & 0,66 & 0,66 \\
NI & 0,50 & 0,75 & 0,86 & 0,50 & 1,00 & 0,67 & 0,71 & 0,33 & 0,33 \\
NW & 0,83 & 1,00 & 0,43 & 0,50 & 1,00 & 0,67 & 0,74 & 0,33 & 0,33 \\
RP & 0,50 & 1,00 & 0,93 & 0,50 & 1,00 & 0,67 & 0,77 & 0,33 & 0,33 \\
SH & 1,00 & 0,50 & 1,00 & 1,00 & 1,00 & 0,67 & 0,86 & 0 & 0,33 \\
SL & 1,00 & 0,25 & 0,50 & 0,50 & 1,00 & 0,00 & 0,54 & 0,66 & 0,66 \\
SN & 0,00 & 0,25 & 0,37 & 0,25 & 1,00 & 0,00 & 0,31 & 0,66 & 0,66 \\
ST & 0,00 & 0,75 & 0,63 & 0,25 & 1,00 & 0,67 & 0,55 & 0,66 & 0,33 \\
TH & 0,92 & 1,00 & 0,58 & 0,50 & 1,00 & 0,67 & 0,78 & 0,33 & 0,33 \\
\hline & & & & & & & & & \\
\end{tabular}

pendix). Als Ergebnis lässt sich festhalten, dass die Migrationspolitik in Berlin und Bremen wenig restriktiv ausfällt, während Bayern die andere Seite des Kontinuums mit einer sehr restriktive Migrationspolitik besetzt. Spiegelbildlich umgekehrt wurden die Outcome-Werte für permissive Migrationspolitik kodiert. Dies gilt ungeachtet der gewählten Kalibrierungsmethode des Outcome. Abweichungen ergeben sich jedoch bei den weniger eindeutigen Fällen: Hessen gehört bei einer Zusammenführung über den Mittelwert zur Gruppe der restriktiven Länder $(0,66)$, gleiches gilt für Sachsen-Anhalt. Beide Länder fallen bei einer eher qualitativ vorgenommenen Einschätzung jedoch in die Gruppe der permissiven Länder (siehe Tab. 8).

Die drei Bedingungen zur Erklärung der migrationspolitischen Varianz zwischen den Bundesländern wurden für die fsQCA-Analyse entlang der theoretischen Vorüberlegungen kalibriert. Dabei wurden die metrisch skalierten Daten den Empfehlungen der Literatur folgend (Schneider und Wagemann 2012) durch direkte Kalibrierung anhand der Feststellung von Ländergruppen und größerer Sprünge in den Verteilungen in die vier fuzzy-Werte überführt (vgl. Tab. 3). Die migrationspolitische Ausrichtung der Landesregierung wurde auf Basis der Daten zur Parteiposition der Landesparteien in der Migrationspolitik von Bräuninger et al. (2020) berechnet, wobei die Positionen aller an der Landesregierung beteiligten Parteien mit den jeweiligen Kabinettssitzen gewichtet wurden. In die Berechnung fanden die beiden letzten Landtagswahlen seit dem Einsetzen der „Flüchtlingskrise“ Eingang, da auch die Messung des Outcome auf diese Zeitperiode abstellt. Die Landesregierung mit der autoritärsten migrationspolitischen Position ist nach dieser Messung mit Abstand die bayerische Staatsregierung, während die Parteien der Regierung im Stadtstaat Bremen die liberalsten Positionen einnahmen (vgl. Appendix, Tab. 11). Die anderen Länder gruppieren sich zwischen diese beiden Pole ein. Zur Untersuchung 
Tab. 3 Kalibrierung der Bedingungen

\begin{tabular}{lllllll}
\hline & $\begin{array}{l}\text { Migrations- } \\
\text { politische } \\
\text { Position der }\end{array}$ & $\begin{array}{l}\text { Kalibrierung } \\
\text { Parteiposi- } \\
\text { tion }\end{array}$ & $\begin{array}{l}\text { Sozio-öko- } \\
\text { nemischer } \\
\text { Misery- } \\
\text { Index }\end{array}$ & $\begin{array}{l}\text { Kalibrierung } \\
\text { Sozioöko- } \\
\text { nomie }\end{array}$ & $\begin{array}{l}\text { Einstellungen } \\
\text { gegenüber } \\
\text { Geflüchteten }\end{array}$ & $\begin{array}{l}\text { Kalibrierung } \\
\text { Einstellun- } \\
\text { gen }\end{array}$ \\
\hline BW & 6,4 & 0 & $-1,05$ & 0 & 0,54 & 0,33 \\
BY & 18,3 & 1 & $-1,26$ & 0 & $-0,02$ & 0,66 \\
BE & 8,0 & 0,33 & 0,78 & 1 & 0,63 & 0,33 \\
BB & 10,1 & 0,33 & 0,38 & 0,66 & $-1,16$ & 1 \\
HB & 5,2 & 0 & 1,07 & 1 & 0,97 & 0 \\
HH & 8,9 & 0,33 & $-0,72$ & 0,33 & 1,01 & 0 \\
HE & 11,0 & 0,66 & $-0,61$ & 0,33 & 0,24 & 0,66 \\
MV & 10,4 & 0,66 & 0,61 & 1 & $-1,08$ & 1 \\
NI & 7,4 & 0,33 & $-0,22$ & 0,66 & 0,85 & 0 \\
NW & 8,4 & 0,33 & 0,11 & 0,66 & 0,92 & 0 \\
RP & 5,9 & 0 & $-0,27$ & 0,66 & 0,64 & 0,33 \\
SL & 11,2 & 0,66 & 0,24 & 0,66 & 0,42 & 0,33 \\
SN & 11,2 & 0,66 & $-0,03$ & 0,66 & $-1,87$ & 1 \\
ST & 12,2 & 0,66 & 0,71 & 1 & $-1,64$ & 1 \\
SH & 7,8 & 0,33 & 0,05 & 0,66 & 0,30 & 0,66 \\
TH & 9,6 & 0,33 & 0,19 & 0,66 & $-1,38$ & 1 \\
\hline
\end{tabular}

der zweiten Hypothese, die davon ausging, dass schlechte sozio-ökonomische Rahmenbedingungen eine notwendige oder hinreichende Bedingung für eine restriktive Migrationspolitik darstellen, wurden die in der Literatur diskutierten Indikatoren Arbeitslosenquote, Verschuldung und Wirtschaftskraft - zwischen 2010 und 2018 gemittelt und über eine z-Standardisierung zu einem Misery-Index zusammengeführt. Dieser weist für die Länder Bayern und Baden-Württemberg die geringsten Werte auf, die wirtschaftlich schlechteste Situation liegt den Zahlen zufolge in Bremen, Berlin, Mecklenburg-Vorpommern und Sachsen-Anhalt vor. Schließlich wurden für die Analyse der dritten Hypothese zu den Einstellungen gegenüber Migranten die Ergebnisse von zwei Fragen einer Politbarometer-Umfrage aus dem Jahr 2017 zum Themenfeld der Migrations- und Asylpolitik herangezogen. ${ }^{12}$ Die Daten bestätigen bereits bekannte Muster - insbesondere zu den Unterschieden in den Bevölkerungseinstellungen zwischen den westdeutschen und den ostdeutschen Bundesländern (vgl. Spalte 6 in Tab. 3): Die Befragten in den fünf ostdeutschen Bundesländern sind deutlich stärker der Meinung, dass Deutschland die aktuellen Flüchtlingszahlen nicht verkraften könne. Zudem sind die Bürger dieser Länder in starkem Maße gegen einen Familiennachzug für geflüchtete Menschen, die in Deutschland einen anerkannten Asylstatus besitzen. Auf der anderen Seite der Ska-

\footnotetext{
12 Es wurden dabei Einstellungen zu Flüchtlingszahlen in Deutschland (Kann Deutschland das verkraften?) sowie zum Familiennachzug (richtig oder falsch?) abgefragt. Beide Fragen wurden unterschiedlich häufig gestellt (Frage 1: N=14.312, Frage 2: N=3426). Für die Analyse wurden die Ergebnisse auf Landesebene aggregiert, z-standardisiert und gemittelt. Da die Daten für Berlin Ost und West gesondert erhoben wurden, haben wir für die Bundeshauptstadt ebenfalls das arithmetische Mittel berechnet.
} 
Tab. 4 Notwendige Bedingungen

\begin{tabular}{lllll}
\hline & \multicolumn{2}{l}{ Outcome: Repressive Migrationspolitik } & \multicolumn{2}{l}{ Outcome: Permissive Migrationspolitik } \\
& Konsistenz & Abdeckung & Konsistenz & Abdeckung \\
\hline $\mathrm{P}$ & 0,810 & 0,895 & 0,443 & 0,631 \\
$\sim \mathrm{P}$ & 0,671 & 0,479 & 0,926 & 0,852 \\
$\mathrm{M}$ & 0,713 & 0,498 & 0,888 & 0,799 \\
$\sim \mathrm{M}$ & 0,718 & 0,822 & 0,450 & 0,663 \\
$\mathrm{E}$ & 0,761 & 0,612 & 0,517 & 0,535 \\
$\sim \mathrm{E}$ & 0,432 & 0,407 & 0,631 & 0,765 \\
\hline
\end{tabular}

$P$ Autoritäre Parteiposition, $M$ hoher Misery-Index, $E$ ablehnende Einstellungen, $\sim P$ nicht autoritäre (liberale) Parteiposition, $\sim M$ nicht hoher Misery-Index, $\sim E$ nicht ablehnende Einstellungen

la sind die Befragten in Bremen, Hamburg, Niedersachsen und Nordrhein-Westfalen vergleichsweise positiv Migranten gegenüber eingestellt.

\section{Bedingungen für restriktive und permissive Migrationspolitik: Ergebnisse der fsQCA-Analyse}

In diesem Abschnitt analysieren wir die Bedingungen für das Vorliegen einer permissiven bzw. restriktiven Migrationspolitik in den deutschen Ländern mithilfe einer fsQCA. Wie in QCA-Analysen üblich, beginnen wir mit einem Test auf notwendige Bedingungen, bevor wir uns der Untersuchung hinreichender Bedingungen und den daraus ableitbaren Kausalpfaden widmen. In beiden Fällen betrachten wir den Outcome permissiver und restriktiver Migrationspolitik, um auch asymmetrische Kausalität identifizieren zu können. Die vorgestellten Ergebnisse entstammen einer Analyse mit der Statistiksoftware R Studio (Dusa 2019).

\subsection{Notwendige Bedingungen}

In einem ersten Schritt überprüfen wir, ob eine der drei Bedingungen als notwendig für das Outcome restriktive bzw. permissive Migrationspolitik betrachtet werden kann. Notwendige Bedingungen bedeuten mengentheoretisch, dass das Outcome eine Untermenge der Bedingung ist - das Outcome also immer dann auftritt wenn auch die Bedingung erfüllt ist. Tab. 4 präsentiert die Ergebnisse der Auswertung. Die Analyse zeigt, dass ausschließlich die Bedingung der nicht-autoritären Parteiideologie ( P) als notwendig für den Outcome der permissiven Migrationspolitik zu werten ist. Die Bedingung hebt sich deutlich von den anderen Konsistenzwerten ab und übertrifft den in der Literatur als Schwellenwert angegebenen Konsistenzwert ${ }^{13}$ von 0,9 (Schneider und Wagemann 2012, S. 143) (siehe kursiv gesetzte Zeile). Zu-

\footnotetext{
13 Der Konsistenzwert gibt an, wie perfekt die Mengenbeziehung zwischen Bedingung und Outcome ist. Je mehr Elemente des Outcomes nicht Teil der Bedingung sind (bzw. je mehr Elemente der Bedingung nicht Teil des Outcomes), umso geringer ist der Konsistenzwert (vgl. Siewert und Wagemann 2018, S. 5).
} 
dem ist der Abdeckungswert mit 0,85 vergleichsweise hoch - d.h. die Bedingung ist sehr relevant für das Auftreten des Outcomes. ${ }^{14}$

Betrachtet man die weiteren Mengenbeziehungen, so fällt auf, dass auch die Bedingung des hohen sozio-ökonomischen Problemdrucks als Übermenge des Outcomes einer permissiven Migrationspolitik einen hohen Konsistenzwert aufweist die Abdeckung ist hier jedoch etwas schlechter. Demnach kann für die Analyse der notwendigen Bedingungen festgestellt werden, dass eine liberale Parteiposition der Regierung eine notwendige Bedingung für permissive Migrationspolitik in den Bundesländern darstellt.

\subsection{Hinreichende Bedingungen}

\subsubsection{Konstruktion der Wahrheitstafel}

Der erste Schritt zur Untersuchung von hinreichenden Bedingungen besteht in der Aufstellung einer Wahrheitstafel (Tab. 5), welche alle möglichen Bedingungskombinationen enthält. In unserem Fall sind dies also bei drei Bedingungen acht mögliche Kombinationen aus Parteiposition der Regierung (P), sozio-ökonomischem Kontext (Misery-Index, M) sowie den Einstellungen der Bevölkerung (E). Ebenfalls aus der Wahrheitstafel ersichtlich sind die empirischen Fälle, welchen mit den jeweiligen Bedingungskonstellationen korrespondieren (letzte Spalte in Tab. 5). Zudem illustriert die Tafel auch die begrenzte empirische Realität, weil drei der möglichen Bedingungskonstellationen (die letzten Zeilen der Tabelle) schlicht nicht durch empirische Fälle abgedeckt werden - in der QCA-Terminologie werden diese als „logical remainders“ bezeichnet. Schließlich kann aus der Wahrheitstafel abgelesen haben, wie konsistent die Mengenbeziehungen sind (Konsistenzwert sowie PRIScore). Anhand dieser Scores kann entschieden werden, welches Outcome den Bedingungskombinationen zugeordnet wird. Um diese Entscheidung zu treffen wird häufig ein Schwellenwert von 0,8 gewählt (Schneider und Wagemann 2012, S. 183) letztlich gilt es aber auf Basis des zugrundeliegenden Samples die Wahl zwischen einem etwas höheren oder niedrigeren Schwellenwert zu treffen. Angesichts des doch deutlichen Unterschieds der Konsistenzwerte zwischen den ersten beiden Bedingungskombinationen (Zeile 1 und 2 in Tab. 5) und den drei anderen Kombinationen mit empirischen Fällen (Zeilen 3-5), liegt es nahe, den „Cut-Off“-Punkt in der Tat bei $0,8 \mathrm{zu}$ setzen. Entsprechend müssten in Tab. 5 für die repressive Migrationspolitik die ersten zwei Zeilen (und die Fälle Bayern, Hessen, Sachsen, Sachsen-Anhalt und Saarland) sowie für den permissive Politik die Zeilen 3-6 (Berlin, Bremen, Niedersachsen, Nordrhein-Westfalen, Rheinland-Pfalz, Brandenburg, Mecklenburg-

\footnotetext{
14 Dies gilt auch für die alternative Kalibrierung des Outcome, auch wenn hier der Konsistenzwert für das Vorliegen einer schlechten sozioökonomischen Lage an den Wert für die Parteiposition heranreicht.

15 Für den alternativen Outcome findet sich die Wahrheitstafel im Appendix. Mit Blick auf die empirischen Fölle unterscheidet sich die Eingruppierung dahingehend, dass Baden-Württemberg und Hamburg in der alternativen Kodierung des Outcomes Teil der permissiven Gruppe sind (d.h. die Zeile 3 in Tab. 5 zu einem permissiven Outcome führt).
} 
Tab. 5 Wahrheitstafel

\begin{tabular}{|c|c|c|c|c|c|c|c|c|c|c|}
\hline & $\mathrm{P}$ & M & $\mathrm{E}$ & $\begin{array}{l}\text { Outcome } \\
\text { restriktiv } \\
(Y)\end{array}$ & Konsist & PRI & $\begin{array}{l}\text { Outcome } \\
\text { permissiv } \\
(\sim Y)\end{array}$ & Konsist & PRI & Fälle \\
\hline 1 & 1 & 0 & 1 & 1 & 0,890 & 0,748 & 0 & 0,662 & 0,246 & BY, HE \\
\hline 2 & 1 & 1 & 1 & 1 & 0,833 & 0,593 & 0 & 0,750 & 0,400 & SL, SN, ST \\
\hline 3 & 0 & 0 & 0 & 0 & 0,787 & 0,308 & 1 & 0,891 & 0,641 & $\mathrm{BW}, \mathrm{HH}$ \\
\hline 4 & 0 & 1 & 1 & 0 & 0,644 & 0,162 & 1 & 0,921 & 0,815 & $\begin{array}{l}\text { BB, MV, } \\
\text { SH, TH }\end{array}$ \\
\hline 5 & 0 & 1 & 0 & 0 & 0,399 & 0,000 & 1 & 0,998 & 0,997 & $\begin{array}{l}\text { BE, HB, } \\
\text { NI, NW, RP }\end{array}$ \\
\hline 6 & 0 & 0 & 1 & \multicolumn{7}{|c|}{ Logical Remainder } \\
\hline 7 & 1 & 0 & 0 & \multicolumn{7}{|c|}{ Logical Remainder } \\
\hline 9 & 1 & 1 & 0 & \multicolumn{7}{|c|}{ Logical Remainder } \\
\hline
\end{tabular}

Vorpommern, Schleswig-Holstein, Thüringen, sowie Baden-Württemberg und Hamburg) kodiert werden. ${ }^{15}$

Im zweiten Schritt gilt es nun durch Minimierung der Wahrheitstafel die kausalen Pfade herauszuarbeiten, welche zu einer restriktiven bzw. permissiven Migrationspolitik führen. Bei Vorliegen von logical remainders - wie in unserem Falle - lassen sich dabei drei Vorgehensweisen unterscheiden. Eine erste Möglichkeit besteht darin, die logical remainders bei der Minimierung der Wahrheitstafel (konservative Lösung) nicht zu betrachten. Dies bedeutet de facto anzunehmen, dass alle empirischen Fälle, die dieser Bedingungskombination entsprechen würden, nicht hinreichend für den Outcome wären - eine starke Vorannahme. Die zweite Möglichkeit ist die der sparsamen Lösung (parsimonious solution), bei der der Lösungsterm möglichst stark vereinfacht wird. Die Nutzung dieser Lösung wird etwa von Thiem (2019) vorgeschlagen. Hier werden also die logical remainders berücksichtigt, welche den Lösungsterm einfacher machen. Schließlich gibt es eine mittlere Strategie (intermediate solution), die auf theoretischen Vorüberlegungen beruht. In unserem Fall sind wir theoretisch von der Erwartung ausgegangen, dass eine autoritäre Parteiposition $(\mathrm{P})$, eine schlechte sozio-ökonomische Lage $(\mathrm{M})$ und negative Einstellungen der Bevölkerung (E) zu einer restriktiveren Migrationspolitik (Y) führen. Diese Vorüberlegungen kann man nun in die Bewertung der logical remainders einfließen lassen (z. B. spricht in Zeile 8 der Wahrheitstafel durch das Vorliegen von P für das Vorliegen von Y) - und damit ggf. eine weitere Vereinfachung des Lösungsterms erreichen. Angesichts der Debatte in der Methodenliteratur berichten wir im Folgenden jeweils die mittlere und die sparsame Lösung und ergänzen die konservative Lösung in einer Fußnote. 
Tab. 6 Hinreichende Bedingungen: restriktive Migrationspolitik

\begin{tabular}{llllll}
\hline & Solution & Konsistenz & Abdeckung & Fälle & \\
\hline Interm & $\mathrm{P} *$ & Kons: 0,824; & 0,952 & $\mathrm{P} * \mathrm{E}: \mathrm{BY}, \mathrm{HE} ; \mathrm{SL}$, & $\sim \mathrm{M}: \mathrm{BW}, \mathrm{HH} ;$ \\
& $\mathrm{E}+\sim \mathrm{M} \rightarrow \mathrm{Y}$ & PRI: 0,648 & & SN, ST & BY, HE \\
Parsim & $\mathrm{P}+\sim \mathrm{M} \rightarrow \mathrm{Y}$ & Kons: 0,824; & 0,952 & $\mathrm{P}: \mathrm{BY}, \mathrm{HE}$; SL, SN, & $\sim \mathrm{M}: \mathrm{BW}, \mathrm{HH} ;$ \\
& & PRI: 0,648 & & $\mathrm{ST}$ & $\mathrm{BY}, \mathrm{HE}$ \\
\hline
\end{tabular}
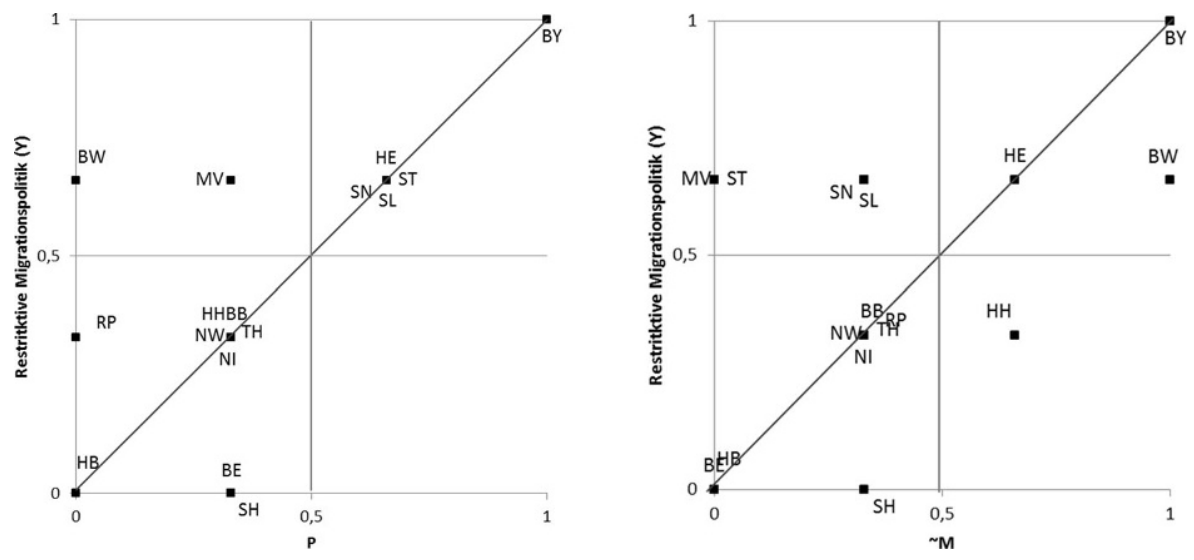

Abb. 2 Hinreichende Bedingungen - restriktive Migrationspolitik

\subsubsection{Hinreichende Pfade für restriktive und permissive Migrationspolitik}

Die Minimierung der Wahrheitstafel für das Outcome der restriktiven Migrationspolitik erbringt bei in allen Varianten eine äquifinale Lösung mit jeweils zwei Pfaden (siehe Tab. 6). ${ }^{16}$

Demzufolge führt eine autoritäre Position der Landesregierung (gepaart mit einer ablehnenden Haltung der Bevölkerung) gegenüber Geflüchteten zu einer restriktiven Migrationspolitik in den Ländern. Dieses Ergebnis bestätigt die Hypothesen 1 und 3 unserer theoretischen Vorüberlegungen. Zudem führt eine gute sozio-ökonomische Position zu einer restriktiveren Politik - diese Erwartung widerspricht unserer theoretischen Vermutung, findet sich aber in allen Lösungspfaden wieder. Eine Minimierung der Wahrheitstafel auf Basis der alternativen Kodierung führt zu ähnlichen Lösungspfaden. Diese sind ebenfalls Kombinationen aus autoritärer Regierungsposition $(\mathrm{P})$, negativen Einstellungen gegenüber Geflüchteten $(\mathrm{E})$ und einer guten sozioökonomischen Lage $(\sim \mathrm{E})$, wobei auch hier die Bedingung $\mathrm{P}$ dominiert (vgl. Appendix, Tab. 15).

Abb. 2 veranschaulicht den gefunden Lösungspfade und die Position der Länder beiden in der sparsamen Lösung gefundenen Pfade. Mit Blick auf die Parteipolitik wird deutlich, dass fünf Bundesländer die erwartete Kombination von restriktiver Politik und autoritärer ideologischer Position der Landesregierung aufweisen (Qua-

16 Conservative solution : $\mathrm{P} * \mathrm{E}+\sim \mathrm{P} * \sim \mathrm{M} * \sim \mathrm{E} \rightarrow \mathrm{Y}$ (Konsistenz: 0,857 ; PRI: 0,683, Abdeckung: 0,903; Fälle: P* E: BY, HE; SL, SN, ST / P * $\sim \mathrm{M} * \sim \mathrm{E}: \mathrm{BW}, \mathrm{HH})$. 
Tab. 7 Hinreichende Bedingungen: permissive Migrationspolitik

\begin{tabular}{|c|c|c|c|c|}
\hline & Solution & Konsistenz & Abdeckung & Fälle \\
\hline $\begin{array}{l}\text { Interm and } \\
\text { Parsim }\end{array}$ & $\sim \mathrm{P} \rightarrow \sim \mathrm{Y}$ & $\begin{array}{l}\text { Kons: } 0,852 \\
\text { PRI: } 0,747\end{array}$ & 0,926 & $\begin{array}{l}\sim \mathrm{P}: \mathrm{BW}, \mathrm{HH} ; \mathrm{NE}, \mathrm{HB}, \mathrm{NI}, \mathrm{NW}, \\
\text { RP; BB, MV, SH }\end{array}$ \\
\hline
\end{tabular}

drant rechts oben). Weitere neun Länder widersprechen den Befunden nicht, da die Parteien an den Landesregierungen eine eher liberale Position in ihren Programmen vertreten und diese auch in permissivere Politik umsetzen. Aus der Reihe tanzt hier nur vor allem Baden-Württemberg - mit einer Landesregierung, die von liberalen programmatischen Positionen (,fully in“) geprägt ist, gleichzeitig jedoch eine eher restriktive Politik betreibt. Ähnlich verhält es sich mit Mecklenburg-Vorpommern, wo eine eher liberale Regierung ebenfalls restriktive Politik umsetzt. Beide Fälle sind für die Betrachtung hinreichender Bedingungen jedoch nicht relevant, da Parteipolitik die Migrationspolitik zwar nicht erklären kann, dafür aber möglicherweise eine andere Bedingung in Frage käme. Alles in allem bestätigt das Diagramm, dass eine autoritäre programmatische Position der Landesregierung eine hinreichende Bedingung für das Auftreten restriktiver Migrationspolitik ist. Mit Blick auf die sozioökonomische Lage ist der Befund weniger eindeutig. Zwar ist die wirtschaftliche Situation in vier Länder mit restriktiver Politik tatsächlich schlecht (MV, ST, SN, SL), gleichzeitig betreiben aber auch drei Länder mit guter Lage restriktive Politik. Besonders relevant ist aber hier der Fall Hamburg, der zur Menge der Länder mit einer guten sozioökonomischen Lage gehört aber eine wenig restriktive Politik betreibt. Erklärt werden kann die Position jedoch über den anderen Lösungspfad und die liberale Regierungsposition.

Analysiert man das Outcome der permissiven Migrationspolitik unterscheiden sich die Pfade für die sparsame und die mittlere Lösung nicht (siehe Tab. 7). ${ }^{17}$

Das Ergebnis der Minimierung bestätigt die starke Rolle der Parteipolitik für die Migrationspolitik der Länder: Eine liberale Regierungsposition scheint nicht nur notwendig, sondern auch hinreichend für eine permissive Politik zu sein - denn die Negation der Bedingung $\mathrm{P}$ taucht in allen Lösungen auf und ist alleine hinreichend für den Outcome in der mittleren und sparsamen Lösung. Wie schon beim Outcome der restriktiven Migrationspolitik ist der Lösungspfad nahezu identisch, wenn wir die alternative Kalibrierung des Outcomes verwenden: Es kommt nur die Bedingung $\mathrm{M}$ hinzu, d.h. eine schlechte sozio-ökonomische Situation (vgl. Tab. 17 im Appendix).

Abb. 3 veranschaulicht die Ergebnisse der QCA-Analyse für permissive Migrationspolitik und stellt den Lösungspfad der ,,intermediate“ und der ,,parsimonious solution“ dar. Insgesamt neun Länder fallen dabei in den Quadranten oben rechts und sind - wie theoretisch erwartet - sowohl Mitglied der Menge ,permissive Migrationspolitik“ als auch der Menge ,liberale Parteiposition der Landesregierung“. Weitere fünf Länder (Bayern, Hessen, Saarland, Sachsen und Sachsen-Anhalt) sind Nicht-Mitglieder in beiden Gruppen. Auffällig sind hingegen die Positionen von Ba-

\footnotetext{
17 Für die konservative Lösung ergeben sich zwei äquifinale Wege: $\sim \mathrm{P} * \sim \mathrm{M}+\sim \mathrm{P} * \sim \mathrm{E} \rightarrow \sim \mathrm{Y}$ (Konsistenz: 0,916; PRI: 0,848; Abdeckung: 0,925); Fälle: $\sim \mathrm{P} * \sim \mathrm{M}$ : BE, HB, NI, NW, RP; BB, MV, SH, TH / P * $\sim$ : BW, HH; BE, HB, NI, NW, RP.
} 
Abb. 3 XY-Diagramm: Hinreichende Bedingungen - permissive Migrationspolitik

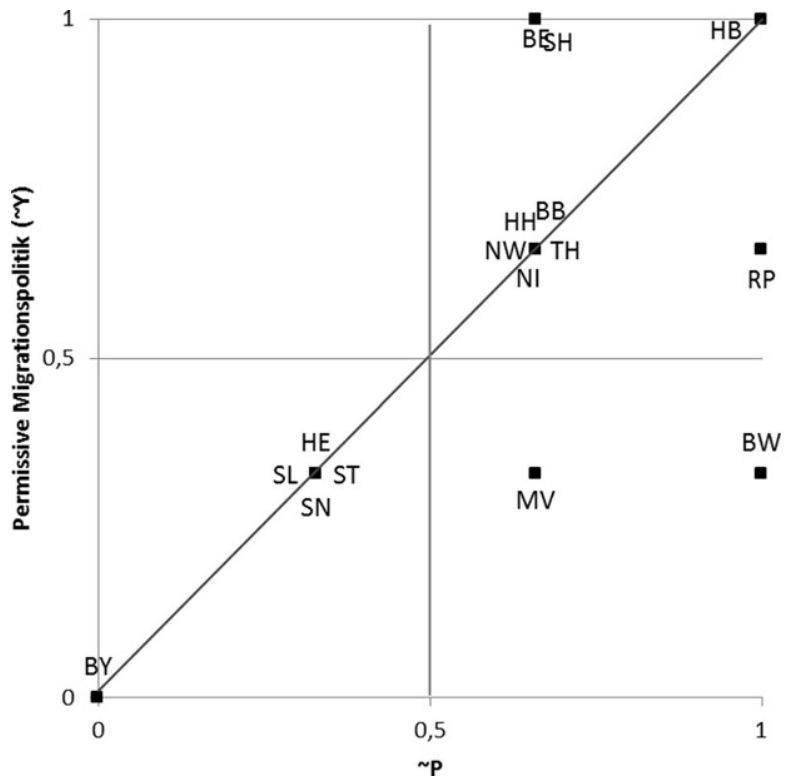

den-Württemberg und, wenn auch etwas weniger deutlich, Mecklenburg-Vorpommern. Beide Länder widersprechen dem Lösungsterm für hinreichende Bedingungen, da zwar Landesregierungen mit eher liberaler Programmatik amtierten, die Migrationspolitik jedoch eher restriktiv ausfiel. Die Sonderposition des Falles BadenWürttemberg lässt sich damit erklären, dass die Regierungen unter Ministerpräsident Kretschmann in einigen Fragen der Migrationspolitik - etwa bei der Abstimmung über die sicheren Herkunftsländer im Bundesrat - eine eher repressive Politik umsetzten, um an anderer Stelle Zugeständnisse zu erreichen (vgl. die Beschreibung im Abschn. 2). Unsere Kodierung ist jedoch „,blind“ für solche „Package Deals“. Zudem ist mit Blick auf die Bedingung der Regierungsposition zu bedenken, dass die starke Regierungsbeteiligung der Grünen zwar den Wert für die Regierungsposition nach oben treibt, das Innenministerium jedoch von der SPD bzw. der CDU gehalten wurde. Die Position Mecklenburg-Vorpommerns als „,contradicting case“ lässt sich wiederum durch die parteipolitische Position plausibilisieren. Diese liegt eher im Mittelfeld der Länder, wurde aber - insbesondere durch einen Schwenk in eine autoritärere Richtung in der zweiten Beobachtungsphase (nach 2016) knapp in die Menge der autoritären Regierungen kodiert (vgl. Tab. 11 im Appendix).

Insgesamt zeigt die Analyse der hinreichenden Bedingungen ein sehr klares Muster, das bereits die Untersuchung der notwendigen Bedingungen dominierte: Die parteipolitische Ausrichtung der Regierung scheint eine zentrale Rolle zu spielen, während die weiteren Bedingungen nur von nachgeordneter Relevanz sind. Zwar tauchen etwa negative Einstellungen gegenüber geflüchteten in zwei der Lösungsterme gemeinsam mit autoritärer Regierungsprogrammatik als Teil einer hinreichenden Lösung auf (vgl. Tab. 6) und die umgekehrte Logik zeigt sich für permissive Politik (vgl. die konservative Lösung in Tab. 7). Angesichts der Dominanz der Parteipolitik würde eine Deutung dieses Ergebnisses aber wohl zu weit führen. Allenfalls für 
eine gute sozio-ökonomische Situation scheinen die Hinweise aus Tab. 6 für einen Zusammenhang zu sprechen, der im Gegensatz zu unseren Erwartungen liegt. In der Gesamtschau dominiert jedoch der Eindruck, dass insbesondere die Parteiprogrammatik der Landesregierung in migrationspolitischen Fragen die Policies stark beeinflusst.

\section{Diskussion und Schlussfolgerungen}

In diesem Aufsatz haben wir uns der Migrationspolitik der Bundesländer aus einer übergreifenden, mehrdimensionalen Perspektive gewidmet und dabei Varianz zwischen den Ländern auf sechs Dimensionen der Migrationspolitik analysiert. Ziel dieses Vorgehens war es, die Literatur, die sich bisher auf einzelne Aspekte von Asylund Migrationspolitik fokussierte, durch eine umfassendere Analyse zu ergänzen. Wir greifen dabei den bestehenden Konzeptualisierungsvorschlag von Reiter und Töller (2019) auf, gehen aber insbesondere durch die Analyse des mehrdimensionalen Outcomes mithilfe einer QCA über den bestehenden Forschungsstand hinaus.

Unsere mehrdimensionale Vermessung der Migrationspolitik erbrachte insgesamt vier Ländergruppen, die mit Blick auf die Permissivität und Restriktivität der politischen Maßnahmen unterschieden werden konnten. Auf Basis dieser Eingruppierung konnten wir hinreichende und notwendige Bedingungen für die Permissivität bzw. die Restriktivität der Migrationspolitik in den deutschen Ländern identifizieren. Die Ergebnisse der fs-QCA zeigen, dass der Einfluss der Parteiprogrammatik eine elementare Rolle spielt: Eine liberale Parteiprogrammatik der Regierung ist eine notwendige Bedingung für permissive Migrationspolitik, gleichzeitig ist die Parteiideologie in nahezu allen Bedingungskonstellationen, die hinreichend für den Outcome sind, in der erwarteten Ausprägung (liberal-permissiv, autoritär-restriktiv) vertreten. Dieses Ergebnis dominiert unsere Untersuchung und findet sich unabhängig von der Kalibrierung des Outcomes.

Eine Abweichung ist hingegen insbesondere für das Bundesland Baden-Württemberg festzustellen (vgl. Abb. 3). Der guten Praxis der qualitative comparative analysis folgend, möchten wir diesen Fall kurz etwas genauer diskutieren. Die Mitgliedschaft Baden-Württembergs in der Menge des restriktiven Outcomes $(0,66)$ bzw. der liberalen Parteiprogrammatik $(0,33)$ lässt sich durch mehrere fallspezifische Erklärungen plausibilisieren. Zum einen ist anzunehmen, dass die zunehmende Hinwendung zu restriktiven Maßnahmen von Baden-Württemberg durch eine Neuzusammensetzung der Regierungskoalition nach den Landtagswahlen 2016 befördert wurde, als die CDU die SPD als Juniorpartnerin in der Koalition mit den Grünen ablöste. Die CDU vertritt aber ein deutlich autoritäreres Asylverständnis als die baden-württembergische SPD (Kostner 2017, S. 192) und übernahm zudem von der SPD das Innenministerium. Dies kann ein weiterer wichtiger Erklärungsfaktor sein, da nach dem „Ministerial-Discretion-Modell“ die Zugehörigkeit des Innenministers zu einer bestimmten Partei den Ausschlag für die Ausgestaltung der Migrationspolitik eines Landes darstellen kann (Töller 2019, S. 580). Ein weiterer Grund ist, dass der grüne Ministerpräsident Kretschmann versuchte in seinem regierungspolitischen Handeln zur Bewältigung der „Flüchtlingskrise“ eine pragmatische 
Linie zu vertreten, die Kompromisse nach sich zog. So hat Baden-Württemberg zwischen Sommer 2014 und Frühherbst 2015 die erste Phase der „Flüchtlingskri-

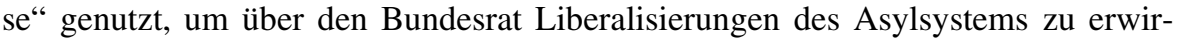
ken. Beispielsweise wurden der Vorrang von Geld- vor Sachleistungen, Zugangserleichterungen zum Arbeitsmarkt und Verkürzungen der Residenzpflicht beschlossen (Kostner 2017, S. 191). Um diese Reformen durchzusetzen, zeigte Baden-Württemberg Kompromissbereitschaft und trug die Einstufung von sechs Balkanstaaten zu sicheren Herkunftsstaaten sowie verschärfte Abschiebungsbestimmungen mit. Für dieses Abweichen von grünen Grundprinzipien wurde der Ministerpräsident BadenWürttembergs Winfried Kretschmann von seiner eigenen Partei stark kritisiert. Der Ministerpräsident selbst betrachtet seine Kompromissbereitschaft hingegen als Beleg für ein auch in Krisenzeiten handlungsfähiges Humanitätsverständnis, welches er als ,pragmatischen Humanismus“ bezeichnet (Kostner 2017, S. 191).

Nicht bestätigen konnten sich zudem die Erwartungen, dass ein hoher sozioökonomischer Problemdruck zu einer restriktiven, bzw. ein niedriger sozioökonomischer Problemdruck zu einer permissiven Migrationspolitik führt. Eine Erklärung hierfür könnte schlicht sein, dass der sozioökonomische Kontext, der laut Theorie auch über die Landeshaushalte relevant werden könnte, auf teilweise sehr verschlungenen Pfaden durch das administrative Dickicht wirkt. Denn die finanziellen Verschränkungen zwischen Ländern und Kommunen sind in der Migrationspolitik landesspezifisch sehr unterschiedlich. Zudem kostet nicht nur permissive Migrationspolitik Geld (etwa die Einführung Gesundheitskarte oder eine dezentrale Unterbringung), sondern auch die von uns als restriktiv eingestufte Einführung von Sachleistungen oder Sicherheitspersonal in den Ankerzentren sind erhebliche Kostenfaktoren. Insofern dürften insbesondere qualitative Studien Aufschluss darüber geben, wie sozio-ökonomische Faktoren auf die Finanzbeziehungen zwischen Bund, Ländern und Kommunen und dadurch vermittelt auf die Migrationspolitik wirken (vgl. etwa Hummel und Thöne 2016).

Schließlich scheinen die Einstellungen der Bevölkerung der Länder kaum eine Rolle zu spielen. Zwar scheinen die Einstellungen in Kombination mit der Parteipolitik als Bedingung in einigen konservativen Lösungstermen der QCA auf, allerdings scheint die Parteipolitik zu dominieren. Dies lässt sich plausibilisieren, wenn wir davon ausgehen, dass Parteiprogramme als Transmissionsriemen wirken und daher die Präferenzen sich über die migrationspolitischen Positionen der Landesparteien abbilden. Wie genau sich Einstellungen der Wähler*innen, Parteiprogrammatik und Regierungspolitik jedoch gegenseitig beeinflussen, bleibt in dieser auf dem Aggregatniveau angesiedelten Studie notwendigerweise im Dunkeln. Die hier zutage geförderten Ergebnisse weisen jedoch darauf hin, dass zukünftige Forschung in den Bundesländern - unabhängig vom Politikfeld - ein stärkeres Augenmerk auf die möglichen Interaktionen zwischen diesen grundlegenden Motivlagen politischer Akteure und deren Auswirkungen auf politische Entscheidungen legen sollte.

Danksagung We want to thank the two reviewers for very helpful comments. We are also grateful that Annette E. Töller has provided very valuable feedback at a much earlier stage of this work.

Funding Open Access funding enabled and organized by Projekt DEAL. 
Open Access Dieser Artikel wird unter der Creative Commons Namensnennung 4.0 International Lizenz veröffentlicht, welche die Nutzung, Vervielfältigung, Bearbeitung, Verbreitung und Wiedergabe in jeglichem Medium und Format erlaubt, sofern Sie den/die ursprünglichen Autor(en) und die Quelle ordnungsgemäß nennen, einen Link zur Creative Commons Lizenz beifügen und angeben, ob Änderungen vorgenommen wurden.

Die in diesem Artikel enthaltenen Bilder und sonstiges Drittmaterial unterliegen ebenfalls der genannten Creative Commons Lizenz, sofern sich aus der Abbildungslegende nichts anderes ergibt. Sofern das betreffende Material nicht unter der genannten Creative Commons Lizenz steht und die betreffende Handlung nicht nach gesetzlichen Vorschriften erlaubt ist, ist für die oben aufgeführten Weiterverwendungen des Materials die Einwilligung des jeweiligen Rechteinhabers einzuholen.

Weitere Details zur Lizenz entnehmen Sie bitte der Lizenzinformation auf http://creativecommons.org/ licenses/by/4.0/deed.de.

Interessenkonflikt D. Meyer, J. Philipp und G. Wenzelburger geben an, dass kein Interessenkonflikt besteht.

\section{Appendix}

\section{Migrationspolitik}

\section{A. Skalierung der Rohdaten}

\section{Anmerkungen zur Skalierung:}

- Gesundheitskarte: Originalskalierung nach dem Index der Bertelsmann-Stiftung (aktualisiert für die Veränderungen nach Mai 2016).

- Ankerzentrum: Qualitative ordinale Kodierung: 0 - Vertrag BMI, viele Zentren; 1 - Vertrag BMI, viele Zentren; 2 - Vertag BMI, anderer Name; 3 - kein Vertrag BMI, Funktionsäquivalente; 4 - Ablehnung Ankerzentren

- Dezentrale Unterbringung: Metrische Werte

- Abschiebung Afganistan: Qualitative ordinale Kodierung: 0 - volle Abschiebung; 1 - Abschiebung Straftäter, Gefährder, Identitätsverweigerer, mit Ausnahmen; 2 - Abschiebung Straftäter, Gefährder, Identitätsverweigerer, ohne Ausnahmen; keine Abschiebung. Die Ausprägung ,keine Abschiebung“ wird hier als qualitativ unterschiedlich von den ersten drei Untergruppen der Kategorie „Abschiebung“ kodiert, und erhält daher den höheren Wert 4.

- Geldleistung/Sachleistung: Dichotome Kodierung: 1 - Geldleistung; 0 - Sachleistung

- Abstimmung Bundesrat: Qualitative ordinale Kodierung: 0 - Zustimmung; 2 Enthaltung; 3 - Ablehnung. Enthaltung wird hier ,näher“" an Ablehnung gezählt, da hierdurch die Mehrheit zum Gesetzentwurf verhindert wird. 
Tab. 8 Skalierung der Rohdaten

\begin{tabular}{lllllll}
\hline & $\begin{array}{l}\text { Gesundheits- } \\
\text { karte }\end{array}$ & $\begin{array}{l}\text { Anker- } \\
\text { zentrum }\end{array}$ & $\begin{array}{l}\text { Dezentrale Un- } \\
\text { terbringung }\end{array}$ & $\begin{array}{l}\text { Abschiebung } \\
\text { Geld- } \\
\text { leistungen }\end{array}$ & $\begin{array}{l}\text { Abstimmung } \\
\text { Bundesrat }\end{array}$ \\
\hline BW & 0 & 4 & 33,9 & 2 & 1 & 0 \\
BY & 0 & 0 & 53,9 & 0 & 0 & 0 \\
BE & 12 & 4 & 60,6 & 2 & 1 & 3 \\
BB & 11 & 4 & 34,4 & 1 & 1 & 2 \\
HB & 10 & 4 & 62,1 & 4 & 1 & 2 \\
HH & 12 & 3 & 88,2 & 2 & 1 & 2 \\
HE & 0 & 3 & 34,2 & 2 & 1 & 2 \\
MV & 0 & 2 & 29,5 & 1 & 1 & 0 \\
NI & 6 & 3 & 77,6 & 2 & 1 & 2 \\
NW & 10 & 4 & 39,4 & 2 & 1 & 2 \\
RP & 6 & 4 & 84,7 & 2 & 1 & 2 \\
SL & 12 & 1 & 45,6 & 2 & 1 & 0 \\
SN & 0 & 1 & 33,7 & 1 & 1 & 0 \\
ST & 0 & 3 & 57,4 & 1 & 1 & 2 \\
SH & 12 & 2 & 90,6 & 4 & 1 & 2 \\
TH & 11 & 4 & 52,3 & 2 & 1 & 2 \\
\hline
\end{tabular}

B. Kalibrierung des Outcomes über den Mittelwert auf 0-1-skalierten Dimensionen

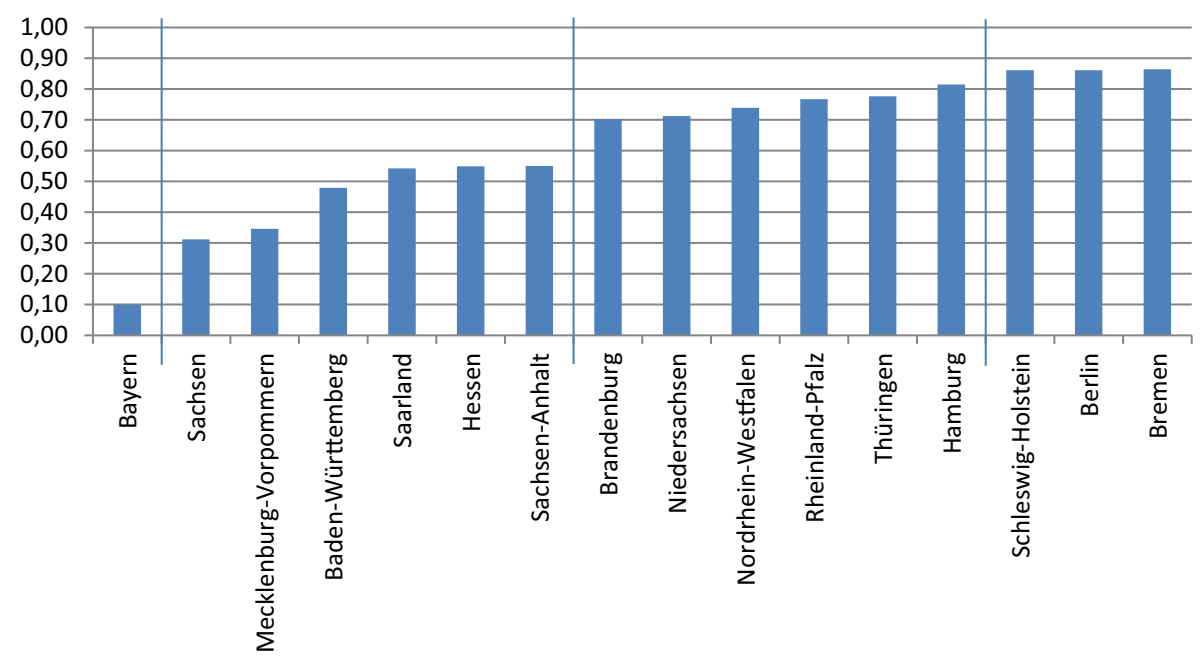

Abb. 4 Kalibrierung Outcome - Schwellenwerte 01-Skalierung 
Tab. 9 Kalibrierung Outcome - 01-Skalierung

\begin{tabular}{lllllllll}
\hline & $\begin{array}{l}\text { Gesund- } \\
\text { heits- } \\
\text { karte }\end{array}$ & $\begin{array}{l}\text { Anker- } \\
\text { zentrum }\end{array}$ & $\begin{array}{l}\text { Dezentrale } \\
\text { Unterbrin- } \\
\text { gung }\end{array}$ & $\begin{array}{l}\text { Abschie- } \\
\text { bung }\end{array}$ & $\begin{array}{l}\text { Geld- } \\
\text { leistungen }\end{array}$ & $\begin{array}{l}\text { Abstimmung } \\
\text { Bundesrat }\end{array}$ & $\begin{array}{c}\text { Mittelwert Fuzzy } \\
\text { Wert }\end{array}$ \\
\hline BB & 0,92 & 1,00 & 0,38 & 0,25 & 1,00 & 0,67 & 0,70 & 0,33 \\
BE & 1,00 & 1,00 & 0,67 & 0,50 & 1,00 & 1,00 & 0,86 & 0 \\
BW & 0,00 & 1,00 & 0,37 & 0,50 & 1,00 & 0,00 & 0,48 & 0,66 \\
BY & 0,00 & 0,00 & 0,59 & 0,00 & 0,00 & 0,00 & 0,10 & 1 \\
HB & 0,83 & 1,00 & 0,69 & 1,00 & 1,00 & 0,67 & 0,86 & 0 \\
HE & 0,00 & 0,75 & 0,38 & 0,50 & 1,00 & 0,67 & 0,55 & 0,66 \\
HH & 1,00 & 0,75 & 0,97 & 0,50 & 1,00 & 0,67 & 0,82 & 0,33 \\
MV & 0,00 & 0,50 & 0,33 & 0,25 & 1,00 & 0,00 & 0,35 & 0,66 \\
NI & 0,50 & 0,75 & 0,86 & 0,50 & 1,00 & 0,67 & 0,71 & 0,33 \\
NW & 0,83 & 1,00 & 0,43 & 0,50 & 1,00 & 0,67 & 0,74 & 0,33 \\
RP & 0,50 & 1,00 & 0,93 & 0,50 & 1,00 & 0,67 & 0,77 & 0,33 \\
SH & 1,00 & 0,50 & 1,00 & 1,00 & 1,00 & 0,67 & 0,86 & 0 \\
SL & 1,00 & 0,25 & 0,50 & 0,50 & 1,00 & 0,00 & 0,54 & 0,66 \\
SN & 0,00 & 0,25 & 0,37 & 0,25 & 1,00 & 0,00 & 0,31 & 0,66 \\
ST & 0,00 & 0,75 & 0,63 & 0,25 & 1,00 & 0,67 & 0,55 & 0,66 \\
TH & 0,92 & 1,00 & 0,58 & 0,50 & 1,00 & 0,67 & 0,78 & 0,33 \\
\hline & & & & & & & & 0,33 \\
\end{tabular}

\section{Kalibrierung des Outcomes über Mengenmitgliedschaften}

\section{Kodierregel für mengenbasierte qualitative Zusammenführung}

- Grundsätzlich ist zu beachten, dass die Dimension „Geldleistungen vs. Sachleistungen“, in der nur Bayern als Mitglied der Menge kodiert werden kann, sehr ungleich verteilt ist.

- Mit 1 kodiert wurde Bayern mit 5 „fully in“ und 1 ,,more out than in“

- Mit 0 kodiert wurden alle Länder, die insgesamt 6 „outs“ erreichen, wobei die vollen Mitgliedschaften mindestens 3 der 6 Dimensionen betreffen. Dies gilt für Berlin (4-2-0-0) und Bremen (3-3-0-0).

- Mit 0,33 kodiert wurden alle sonstigen Länder, bei denen die „outs“ überwiegen: Brandenburg, Hamburg, Hessen, Niedersachsen, Nordrhein-Westfalen, Rheinland-Pfalz, Sachsen-Anhalt und Schleswig-Holstein.

- Mit 0,66 kodiert wurden alle sonstigen Länder, bei denen die ,ins“ überwiegen: Mecklenburg-Vorpommern und Sachsen

- Bei zwei Ländern sind ,,ins“ und „outs“ insgesamt ausgeglichen: Baden-Württemberg und Saarland. Beide Länder wurden als ,,more in than out“ kodiert, da es insgesamt schwieriger ist, 6 ,ins“ zu erreichen, weil nur in Bayern Sachleistungen statt Geldleistungen verteilt werden. Zusätzlich überwiegen im Fall BadenWürttemberg die ,ins“ ebenfalls, da hier drei ,fully ins“ zwei ,fully outs“ gegenüberstehen. 


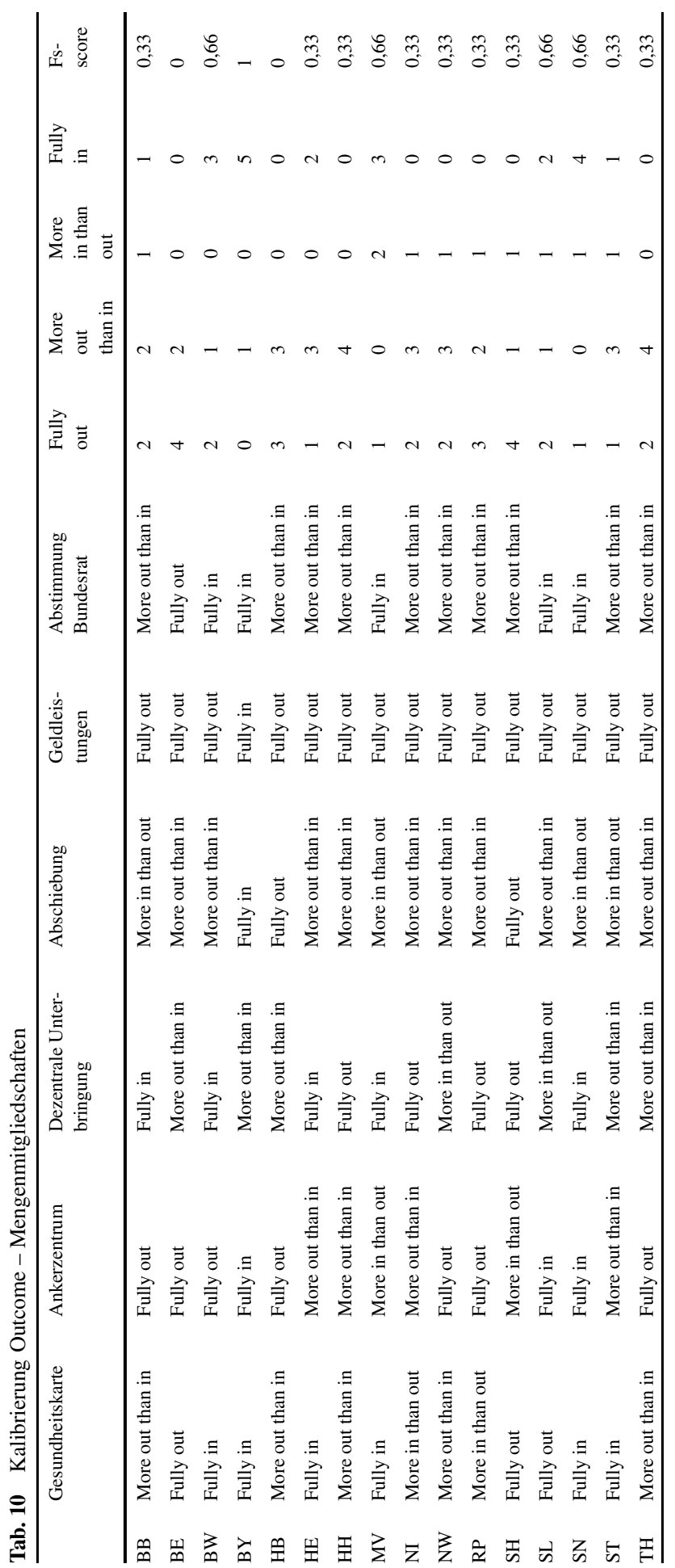




\section{Kalibrierung der Bedingungen}

Tab. 11 Kalibrierung Parteiposition

\begin{tabular}{llll}
\hline & Regierungsposition & Delta & Kalibrierung \\
\hline HB & 5,23 & - & 0 \\
RP & 5,92 & 0,69 & 0 \\
BW & 6,36 & 0,44 & 0 \\
NI & $\mathbf{7 , 4 3}$ & $\mathbf{1 , 0 7}$ & $\mathbf{0 , 3 3}$ \\
SH & 7,79 & 0,36 & 0,33 \\
BE & 7,97 & 0,18 & 0,33 \\
NW & 8,36 & 0,39 & 0,33 \\
HH & 8,91 & 0,55 & 0,33 \\
TH & 9,56 & 0,65 & 0,66 \\
BB & 10,11 & 0,55 & 0,66 \\
MV & 10,36 & 0,25 & 0,66 \\
HE & $\mathbf{1 1 , 0 3}$ & $\mathbf{0 , 6 7}$ & $\mathbf{0 , 6 6}$ \\
SL & 11,18 & 0,15 & 0,66 \\
SN & 11,18 & 0,00 & 0,66 \\
ST & 12,18 & 1,00 & 0,66 \\
BY & $\mathbf{1 8 , 2 9}$ & $\mathbf{6 , 1 1}$ & $\mathbf{1}$ \\
\hline
\end{tabular}

Der Schwellenwert wurde hier bei Hessen gesetzt (und nicht bei Thüringen), da qualitativ die Regierung Ramelow während der entscheidenden Phase der Migrationspolitik im Rahmen der Ankunft der Geflüchteten in Deutschland amtierte (seit Dezember 2014)

Tab. 12 Kalibrierung sozio-ökonomische Lage

\begin{tabular}{llll}
\hline & Misery-Index & Delta & Kalibrierung \\
\hline BY & $-1,26$ & - & 0 \\
BW & $-1,05$ & 0,21 & 0 \\
HH & $\mathbf{- 0 , 7 2}$ & 0,33 & $\mathbf{0 , 3 3}$ \\
HE & $-0,61$ & 0,11 & 0,33 \\
RP & $\mathbf{- 0 , 2 7}$ & 0,34 & $\mathbf{0 , 6 6}$ \\
NI & $-0,22$ & 0,05 & 0,66 \\
SN & $-0,03$ & 0,20 & 0,66 \\
SH & 0,05 & 0,08 & 0,66 \\
NW & 0,11 & 0,06 & 0,66 \\
TH & 0,19 & 0,08 & 0,66 \\
SL & 0,24 & 0,05 & 0,66 \\
BB & 0,38 & 0,14 & 0,66 \\
MV & $\mathbf{0 , 6 1}$ & 0,23 & $\mathbf{1}$ \\
ST & 0,71 & 0,10 & 1 \\
BE & 0,78 & 0,07 & 1 \\
HB & 1,07 & 0,29 & 1 \\
\hline
\end{tabular}


Tab. 13 Kalibrierung Einstellungen der Bevölkerung gegenüber Geflüchteten

\begin{tabular}{llll}
\hline & Einstellungen & Delta & Kalibrierung \\
\hline SN & 54,25 & - & 1 \\
ST & 56,05 & 1,80 & 1 \\
TH & 58,00 & 1,95 & 1 \\
BB & 59,75 & 1,75 & 1 \\
MV & 60,35 & 0,60 & 1 \\
BY & $\mathbf{6 8 , 3 5}$ & 8,00 & $\mathbf{0 , 6 6}$ \\
HE & 70,35 & 2,00 & 0,66 \\
SH & 70,85 & 0,50 & 0,66 \\
SL & 71,65 & 0,80 & 0,66 \\
BW & $\mathbf{7 2 , 7 0}$ & 1,05 & $\mathbf{0 , 3 3}$ \\
BE & 73,45 & 0,75 & 0,33 \\
RP & 73,55 & 0,10 & 0,33 \\
NI & $\mathbf{7 5 , 0 5}$ & 1,50 & $\mathbf{0}$ \\
NW & 75,60 & 0,55 & 0 \\
HB & 75,85 & 0,25 & 0 \\
HH & 76,35 & 0,50 & 0 \\
\hline
\end{tabular}

\section{E. QCA mit alternativem Outcome (Kalibrierung über Mengenmitgliedschaften)}

Tab. 14 Notwendige Bedingungen (alternativer Outcome)

\begin{tabular}{lllll}
\hline & \multicolumn{2}{l}{ Outcome: Repressive Migrationspolitik } & \multicolumn{2}{l}{ Outcome: Permissive Migrationspolitik } \\
& Konsistenz & Abdeckung & Konsistenz & Abdeckung \\
\hline $\mathrm{P}$ & 0,800 & 0,842 & 0,499 & 0,736 \\
$\sim \mathrm{P}$ & 0,752 & 0,511 & 0,895 & 0,853 \\
$\mathrm{M}$ & 0,749 & 0,498 & 0,893 & 0,832 \\
$\sim \mathrm{M}$ & 0,753 & 0,822 & 0,470 & 0,718 \\
$\mathrm{E}$ & 0,749 & 0,574 & 0,570 & 0,612 \\
$\sim \mathrm{E}$ & 0,502 & 0,450 & 0,610 & 0,767 \\
\hline
\end{tabular}

$P$ Autoritäre Parteiposition, $M$ hoher Misery-Index, $E$ ablehnende Einstellungen, $\sim P$ nicht autoritäre (liberale) Parteiposition, $\sim M$ nicht hoher Misery-Index, $\sim E$ nicht ablehnende Einstellungen 
Tab. 15 Wahrheitstafel (alternativer Outcome)

\begin{tabular}{|c|c|c|c|c|c|c|c|c|c|c|}
\hline & $\mathrm{P}$ & M & $\mathrm{E}$ & $\begin{array}{l}\text { Outcome } \\
\text { restriktiv } \\
(Y)\end{array}$ & Konsist & PRI & $\begin{array}{l}\text { Outcome } \\
\text { permissiv } \\
(\sim Y)\end{array}$ & Konsist & PRI & Fälle \\
\hline 1 & 1 & 0 & 1 & 1 & 0,890 & 0,667 & 0 & 0,773 & 0,320 & BY, HE \\
\hline 2 & 0 & 0 & 0 & 0 & 0,882 & 0,444 & 0 & 0,893 & 0,471 & $\mathrm{BW}, \mathrm{HH}$ \\
\hline 3 & 1 & 1 & 1 & 0 & 0,833 & 0,492 & 0 & 0,833 & 0,496 & SL, SN, ST \\
\hline 4 & 0 & 1 & 1 & 0 & 0,712 & 0,193 & 1 & 0,932 & 0,782 & $\begin{array}{l}\mathrm{BB}, \mathrm{MV}, \mathrm{SH}, \\
\mathrm{TH}\end{array}$ \\
\hline 5 & 0 & 1 & 0 & 0 & 0,465 & 0,000 & 1 & 0,998 & 0,996 & $\begin{array}{l}\text { BE, HB, NI, } \\
\text { NW, RP }\end{array}$ \\
\hline 6 & 0 & 0 & 1 & \multicolumn{7}{|c|}{ Logical Remainder } \\
\hline 7 & 1 & 0 & 0 & \multicolumn{7}{|c|}{ Logical Remainder } \\
\hline 9 & 1 & 1 & 0 & \multicolumn{7}{|c|}{ Logical Remainder } \\
\hline
\end{tabular}

Die Schwellenwerte zum Outcome sind eindeutig für die Trennung der ersten Zeile von den Zeilen 4 und 5. Für die Zeilen 2 und 3 liegen in dieser alternativen Kodierung simultane Untermengenbeziehungen vor, insgesamt sind die Aussagen hier inkonklusiv (Schneider und Wagemann 2012, S. 233-247; Siewert 2017, S. 291). Wir folgen hier einer konservativen Strategie, die angesichts der sehr unklaren Zuordnung der Zeilen angebracht erscheint, und schlagen die Bedingungskombinationen in den Zeilen 2 und 3 weder dem restriktiven noch dem permissiven Outcome zu

Tab. 16 Lösungspfade restriktive Migrationspolitik (alternativer Outcome)

\begin{tabular}{lllll}
\hline & Solution & Konsistenz & Abdeckung & Fälle \\
\hline Cons \& & $\mathrm{P} * \sim \mathrm{M} *$ & Kons: 0,890; PRI: 0,667 & 0,402 & BY, HE \\
Interm & $\mathrm{E} \rightarrow \mathrm{Y}$ & & & \\
Parsim & $\mathrm{P} *$ & $\mathrm{P} * \sim \mathrm{M}:$ Kons: 0,924; PRI: 0,575 & $\mathrm{P} * \sim \mathrm{M}: 0,694$ & $\mathrm{P} * \sim \mathrm{M}: \mathrm{BY}, \mathrm{HE}$ \\
& $\sim \mathrm{M}+\sim \mathrm{M} *$ & $\sim \mathrm{M} * \mathrm{E}:$ Kons: 0,902; PRI: 0,647 & $\sim \mathrm{M} * \mathrm{E}: 0,502$ & $\sim \mathrm{M} * \mathrm{E}: \mathrm{BY}, \mathrm{HE}$ \\
& $\mathrm{E} \rightarrow \mathrm{Y}$ & & & \\
\hline
\end{tabular}

Tab. 17 Lösungspfade permissive Migrationspolitik (alternativer Outcome)

\begin{tabular}{lllll}
\hline & Solution & Konsistenz & Abdeckung & Fälle \\
\hline Cons \& Interm & $\sim \mathrm{P}^{*}$ & Kons: 0,955 & 0,823 & BE, HB, NI, NW, RP; BB, \\
$\&$ Parsim & $\mathrm{M} \rightarrow \sim \mathrm{Y}$ & PRI: 0,909 & & MV, SH, TH \\
\hline
\end{tabular}

\section{F. Datenquellen}

\section{Outcome}

- Gesundheitskarte: Bertelsmann-Stiftung (Wächter-Raquet 2016) und eigene Ergänzungen

- Ankerzentren: Eigene Auswertung auf Basis von Reiter und Töller (2019).

- Dezentrale Unterbringung: Statistisches Bundesamt: Tab. 22221-0011. Empfänger von Regelleistungen nach dem Asylbewerberleistungsgesetz: Bundesländer, Stichtag, Art der Unterbringung. Zugriff unter: https://www-genesis.destatis.de/ genesis/online/link/tabellen/22221, zu-letzt geprüft am 24.07.2019.

- Abschiebung Afghanistan: Eigene Auswertung auf Basis von Reiter und Töller (2019). 
- Geldleistungen: Eigene Auswertung

- Abstimmung ,sichere Herkunftsländer“ im Bundesrat: Eigene Auswertung unter Rückgriff auf die Dokumentation des Abstimmungsverhaltens der Länder im Bundesrat zu dem Gesetzesentwurf zur Einstufung von sicheren Herkunftsstaaten am 10.03.2017.

\section{Bedingungen}

- Parteiposition: Datenspende des Mannheimer Teams (Bräuninger et al. 2020).

- Sozioökonomische Situation: Arithmetisches Mittel der z-standarisierten Werte zu Arbeitslosigkeit, Verschuldung pro Kop und BIP pro Kopf (jeweils auf Landesebene). Datenquelle: Statistisches Bundesamt (Genesis-Datenbank).

- Einstellungen der Bevölkerung gegenüber Geflüchteten: Mittelwert der Antworten auf zwei Fragen, die im Politbarometer in 2017 gestellt wurden.

1. „Flüchtlingszahlen in Deutschland: Kann Deutschland das verkraften?“

2. „Einstellung zum Familiennachzug, richtig oder falsch?“

Datenquelle ist das Politbarometer (erreicht über die Gesis-Zacat-Datenbank).

\section{Literatur}

Allport, Gordon W. 1954. The nature of prejudice. Cambridge: Addison-Wesley.

Becker, Julia C., Inna Ksenofontov, Angelika Love, und Lisa Borgert. 2018. Einstellungen und Verhaltensweisen gegenüber Geflüchteten. In Beiträge zur Angewandten Psychologie - Die Flüchtlingsdebatte in Deutschland - Sozialpsychologische Perspektiven, Hrsg. Anette Rohmann, Stefan Stürmer. Berlin: Peter Lang.

Behnke, Nathalie. 2019. Wer zahlt für die Flüchtlinge? Praktische Umsetzung des Konnexitätsprinzips. In Verwaltungshandeln in der Flüchtlingskrise, Hrsg. Jörg Bogumil, Sabine Kuhlmann, und Isabelle Proeller, 65-82.

Bogumil, Jörg, Sabine Kuhlmann, und Isabella Proeller (Hrsg.). 2019. Verwaltungshandeln in der Flüchtlingskrise. Baden-Baden: Nomos.

Bräuninger, Thomas, Marc Debus, Jochen Müller, und Christian Stecker. 2020. Parteienwettbewerb in den deutschen Bundesländern. Wiesbaden: Springer VS.

Bundesministerium des Innern, für Bau und Heimat. 2018. Masterplan Migration - Maßnahmen zur Ordnung, Steuerung und Begrenzung der Zuwanderung. https://www.bmi.bund.de/SharedDocs/ downloads/DE/veroeffentlichungen/themen/migration/masterplan-migration.pdf?_blob=publication File\&v=7. Zugegriffen: 31. Okt. 2019.

Consterdine, Erica, und James Hampshire. 2019. Convergence, capitalist diversity, or political volatility? Immigration policy in Western Europe. Journal of European Public Policy https://doi.org/10.1080/ 13501763.2019.1674364.

de Wenden, Catherine Wihtol. 2017. Actual patterns of migration flows: the challenge of migration and asylum in contemporary europe. In Solidarity in the European Union: a fundamental value in crisis, Hrsg. Andreas Grimmel, Susanne My Giang, 67-79. Cham: Springer.

Dusa, Adrian. 2019. QCA with R. A comprehensive resource. Cham: Springer.

FAZ (Hrsg.). 2019. Sichere Herkunftsländer - Bundesrat vertagt Abstimmung. https://www.faz.net/ aktuell/politik/inland/sichere-herkunftslaender-bundesrat-hat-abstimmung-vertagt-16042507.html. Zugegriffen: 15. Febr. 2019.

Geddes, Andrew. 2018. The politics of European Union migration governance. JCMS: Journal of Common Market Studies 56(S1):120-130.

Gerl, Maximilian, und Yannic Rehm. 2016. Sachleistungen für Flüchtlinge - Selbst in Bayern klappt es ohne Bargeld nicht. In Der Spiegel (20. März 2016). https://www.spiegel.de/politik/deutschland/ sachleistungen-fuer-fluechtlinge-selbst-in-bayern-klappt-es-nicht-a-1082282.html. Zugegriffen: 29. Juli 2019. 
GKV-Spitzenverband (Hrsg.). 2016. Asylbewerber / Flüchtlinge. https://www.gkv-spitzenverband.de/ presse/themen/fluechtlinge_asylbewerber/fluechtlinge.jsp. Zugegriffen: 21. Juli 2019.

Günther, Wolfgang, Dennis Kurrek, und Annette Elisabeth Töller. 2019. Ein starker Fall für die Parteiendifferenztheorie: Die Einführung der Gesundheitskarte für Asylsuchende in den Bundesländern. Zeitschrift für Politikwissenschaft 29(3):361-392.

Helbling, Marc, und Dorina Kalkum. 2018. Migration policy trends in OECD countries. Journal of European Public Policy 25(12):1779-1797.

Henkes, Christian. 2008. Integrationspolitik in den Bundesländern? In Die Politik der Bundesländer: Staatstätigkeit im Vergleich, Hrsg. Achim Frieder Wolf Hildebrandt, 113-135. Wiesbaden: VS.

Hildebrandt, Achim, und Frieder Wolf. 2016. Politik in den Bundesländern unter reformierten institutionellen Rahmenbedingungen. In Die Politik der Bundesländer: Zwischen Föderalismusreform und Schuldenbremse, Hrsg. Achim Frieder Wolf Hildebrandt, 1-9. Wiesbaden: VS.

Hörisch, Felix. 2018. Asylpolitik im Bundesländervergleich. Zeitschrift für Vergleichende Politikwissenschaft 12(4):783-803.

Hörisch, Felix, und Matthias Heiken. 2018. Qualitative Comparative Analysis. Mit einer Anwendung zur Asylbewerberunterbringung im Bundesländervergleich. In Fortgeschrittene Analyseverfahren in den Sozialwissenschaften, Hrsg. Markus Tausendpfund, 203-228. Hagen: Fernuniversität.

Hummel, Caroline-Antonia, und Michael Thöne. 2016. Finanzierung der Flüchtlingspolitik. Für eine ausgewogene Finanzierung der Flüchtlingsleistungen bei Bund, Ländern und Kommunen. Fifo-Berichte Nr. 21, Februar 2016. Köln: Finanzwissenschaftliches Forschungsinstitut an der Universität zu Köln.

Kaiser, Susanne. 2015. Was brauchen Flüchtlinge zum Leben? In Zeit Online (29. Oktober 2015). https:// www.zeit.de/wirtschaft/2015-10/asylpolitik-sachleistungen-bundeslaender. Zugegriffen: 29. Juli 2019.

Kieler Nachrichten (Hrsg.). 2019. Neumünster wird zum Ankunftszentrum. https://www.kn-online.de/ Nachrichten/Schleswig-Holstein/Seehofer-und-Grote-unterzeichnen-Vertrag-fuer-Ankunftszentrumin-Kiel. Zugegriffen: 13. Juni 2019.

Kostner, Sandra. 2017. Asyl und Integration: Aufbrüche in stürmischen Zeiten. In Das grün-rote Experiment in Baden-Württemberg - Eine Bilanz der Landesregierung Kretschmann 2011-2016, Hrsg. Felix Hörisch, Stefan Wurster, 185-222. Wiesbaden: VS.

Kotzur, Patrick. 2018. Kontakt zwischen Geflüchteten und Einheimischen - wie kann man für die Entspannung von Beziehungen zwischen verschiedenen Bevölkerungsgruppen sorgen? http:// www.fachnetzflucht.de/kontakt-zwischen-gefluechteten-undeinheimischen-wie-kann-man-fuerdie-entspannung-von-beziehungen-zwischen-verschiedenen-bevoelkerungsgruppen-sorgen/. Z Zugegriffen: 5. Okt. 2019.

Landmann, Helen Anna Lisa Aydin. Rolf van Dick, und Ulrich Klocke. 2018. Die Kontakthypothese - Wie Kontakt Vorurteile reduzieren und Integration Geflüchteter fördern kann. https:// www.researchgate.net/publication/318318442_Die_Kontakthypothese_Wie_Kontakt_Vorurteile_ gegenuber_Gefluchteten_reduzieren_und_Integration_fordern_kann_The_contact_hypothesis_How_ contact_can_reduce_prejudice_towards_refugees_and_improve_integrat/related. Zugegriffen: 5 . Okt. 2019.

Müller, Wolfgang C., und Kaare Strøm. 1999. Policy, Office, Or Votes?: How Political Parties in Western Europe Make Hard Decisions. Cambridge: Cambridge University Press.

NDR (Hrsg.). 2019. Schnellere Asylverfahren ohne „Ankerzentrum“. https://www.ndr.de/nachrichten/ mecklenburg-vorpommern/Schnellere-Asylverfahrenohne-Ankerzentrum, asylverfahren122.html. Zugegriffen: 9. Apr. 2019.

Pettigrew, Thomas F., und Linda R. Tropp. 2006. A meta-analytic test of intergroup contact theory. Journal of Personality and Social Psychology 90(5):751-783.

Radtke, Ina, und Thurid Hustedt. 2019. Die Flüchtlingskrise als Herausforderung für die interministerielle Koordination in den Bundesländern - das Fallbeispiel Rheinland-Pfalz. In Verwaltungshandeln in der Flüchtlingskrise, Hrsg. Jörg Bogumil, Sabine Kuhlmann, und Isabella Proeller, 49-64. Baden-Baden: Nomos.

Ragin, Charles. 1987. The Comparative Method. Moving beyond qualitative and quantitative strategies. Berkeley, Los Angeles, London: University of California Press.

Reiter, Renate, und Annette E. Töller. 2019. Permissive und restriktive Muster in den Asylpolitiken der Bundesländer. dms - der moderne staat - Zeitschrift für Public Policy, Recht und Management 12(1):194-222.

Riedel, Lisa, und Gerald Schneider. 2017. Dezentraler Asylvollzug diskriminiert: Anerkennungsquoten von Flüchtlingen im bundesdeutschen Vergleich, 2010-2015. PVS Politische Vierteljahresschrift 58(1):23-50. 
Röhlung, Lisa-Marie, und Michel Rabba. 2018. In Bremen inhaftierter Afghane - Geplante Abschiebung sorgt für Kritik. https://www.weser-kurier.de/bremen/bremen-stadt_artikel,-geplante-abschiebungsorgt-fuer-kritik-_arid, 1772711.html. Zugegriffen: 31. Okt. 2019.

Sack, Detlef, und Annette E. Töller. 2018. Einleitung: Policies in den deutschen Ländern. Zeitschrift für Vergleichende Politikwissenschaft 12(4):603-619.

Schammann, Hannes. 2015. Wenn Variationen den Alltag bestimmen. Unterschiede lokaler Politikgestaltung in der Leistungsgewährung für Asylsuchende. Zeitschrift für Vergleichende Politikwissenschaft 9(3):161-182.

Schammann, Hannes. 2019. Migrationspolitik im Mehrebenensystem. In Verwaltungshandeln in der Flüchtlingskrise, Hrsg. Jörg Bogumil, Sabine Kuhlmann, und Isabelle Proeller, 27-42.

Schammann, Hannes. 2018. Ankerzentren werden ihre selbst gesteckten Ziele verfehlen. https://www. fes.de/themenportal-flucht-migration-integration/artikelseite-flucht-migration-integration/anker-ze ntren-werden-ihre-selbst-gesteckten-ziele-verfehlen. Zugegriffen: 6. Nov. 2019.

Schmidt, Manfred G. 1993. Theorien der international vergleichenden Staatstätigkeitsforschung. In PolicyAnalyse. Kritik und Neuorientierung PVS-Sonderheft 24., Hrsg. Adrienne Héritier, 371-393. Opladen: Westdeutscher Verlag.

Schneider, Carsten Q., und Claudius Wagemann. 2012. Set-theoretic methods for the social sciences. Cambridge: Cambridge University Press.

Siewert, Markus B. 2017. Qualitative Comparative Analysis. In Neue Trends in den Sozialwissenschaften: Innovative Techniken für qualitative und quantitative Forschung, Hrsg. Sebastian Jäckle, 273-305. Wiesbaden: Springer.

Siewert, Markus, und Claudius Wagemann. 2018. Qualitative Comparative Analysis. In Handbuch Methoden der Politikwissenschaft, Hrsg. Achim Goerres, Markus Siewert, und Claudius Wagemann, 1-29. Wiesbaden: VS.

Spiegel Online (Hrsg.). 2017. Mehrere Bundesländer stellen Abschiebungen nach Afghanistan zurück. https://www.spiegel.de/politik/deutschland/fluechtlinge-mehrere-bundeslaender-stellen-abschiebun gen-nach-afghanistan-zurueck-a-1133281.html. Zugegriffen: 6. Febr. 2017.

Stukenberg, Timo. 2017. Flüchtlinge sind im teuren „Fehlbeleger“-Status gefangen. In Welt (16. Oktober 2017). https://www.welt.de/finanzen/immobilien/article169666950/Fluechtlinge-sind-im-teurenFehlbeleger-Status-gefangen.html. Zugegriffen: 31. Okt. 2019.

Stürmer, Stefan. 2008. Die Kontakthypothese. In Stereotype, Vorurteile und soziale Diskriminierung. Theorien, Befunde und Interventionen, Hrsg. Lars Eric Petersen, Bernd Six, 283-291. Weinheim: Beltz.

Stuttgarter Zeitung (Hrsg.). 2018. Streit um ,sichere Herkunftsstaaten“. https://www.stuttgarter-zeitung. de/inhalt.asyldebatte-spaltet-landesregierung-streit-um-sichere-herkunftsstaaten.2a58b588-2f5842fb-add1-6fd8361663c7.html. Zugegriffen: 18. Juli 2018.

Süddeutsche Zeitung (Hrsg.). 2019. Strobl will Abschiebungen nach Afghanistan. https://www. sueddeutsche.de/news/politik/migration---stuttgart-strobl-will-abschiebungen-nach-afghanistangruene-dagegen-dpa.urn-newsml-dpa-com-20090101-190612-99-613080. Zugegriffen: 12. Juni 2019.

Tagesschau (Hrsg.). 2018. Vertraulicher Afghanistan-Bericht - Bedrohung durch Taliban und IS bleibt. https://www.tagesschau.de/inland/vertraulicher-bericht-afghanistan-101.html. Zugegriffen: 1. Juni 2018.

Tagesschau (Hrsg.). 2019. Pilotprojekt Ankerzentren - „Es geht doch nur um einen Namen“. https://www. tagesschau.de/inland/asylpolitik-ankerzentren-101.html. Zugegriffen: 2. Febr. 2019.

Tagesspiegel (Hrsg.). 2018. Flüchtlingspolitik - Berliner SPD fällt Bundesvorsitzenden Nahles in den Rücken. https://www.tagesspiegel.de/politik/fluechtlingspolitikberliner-spd-faellt-bundesvorsitzen der-nahles-in-den-ruecken/22995816.html. Zugegriffen: 4. Sept. 2018.

Thiem, Alrik. 2019. Beyond the Facts: Limited Empirical Diversity and Causal Inference in Qualitative Comparative Analysis. Sociological Methods \& Research. https://doi.org/10.1177/00491241198824 63.

Thränhardt, Dietrich. 2001. Zuwanderungs- und Integrationspolitik in föderalistischen Ländern. In Integrationspolitik in föderalistischen Systemen, Hrsg. Lale Akgün, Dietrich Thränhardt, 15-34. Münster: LIT.

Töller, Annette E. 2019. Kein Grund zum Feiern! Die Umwelt- und Energiepolitik der dritten Regierung Merkel (2013-2017). In Zwischen Stillstand, Politikwandel und Krisenmanagement. Eine Bilanz der Regierung Merkel 2013-2017, Hrsg. Reimut Zohlnhöfer, Thomas Saalfeld, 569-590. Wiesbaden: VS.

Wächter-Raquet, Marcus. 2016. Einführung der Gesundheitskarte für Asylsuchende und Flüchtlinge. Der Umsetzungsstand im Überblick der Bundesländer. Gütersloh: Bertelsmann Stiftung. https://www. 
bertelsmannstiftung.de/fileadmin/fles/BSt/Publikationen/GrauePublikatonen/Studie_VV_Gesundhei tskarte_Fluechtlinge_2016.pdf. Zugegriffen: 21. Juli 2019.

Wagemann, Claudius. 2015. Qualitative Comparative Analysis. In Handbuch Policy-Forschung, Hrsg. Georg Wenzelburger/Reimut Zohlnhöfer, 429-452. Wiesbaden: Springer VS.

Wendel, Kay 2014. Unterbringung von Flüchtlingen in Deutschland. Regelungen und Praxis der Bundesländer im Vergleich. Frankfurt a.M.: PRO Asyl. Verfügbar unter: https://www.proasyl.de/wpcontent/ uploads/2015/04/Laendervergleich_Unterbrin-gung_2014-0923_01.pdf. Zugegriffen: 21. Juli 2019.

Wenzelburger, Georg. 2015. Parteien. In Handbuch Policy-Forschung, Hrsg. Georg Wenzelburger, Reimut Zohlnhöfer, 81-112. Wiesbaden: VS.

Wenzelburger, Georg, und Reimut Zohlnhöfer. 2015. Handbuch Policy-Forschung. Wiesbaden: Springer VS.

Wenzelburger, Georg, Stefan Wurster, und Markus B. Siewert. 2020. Responsive Politikgestaltung in den deutschen Bundesländern? Versuch einer Systematisierung und Konzeption eines Forschungsprogramms. Zeitschrift für Vergleichende Politikwissenschaft 14:33-47. https://doi.org/10.1007/s12286020-00449-2.

Wolf, Frieder. 2011. Die Einbindung zivilgesellschaftlicher Akteure in die Härtefallkommissionen der Bundesländer: Bandbreite, Ursachen, Folgen. Zeitschrift für Politikwissenschaft 21(2):259-291.

Zeit Online (Hrsg.). 2019. Keine weiteren Ankerzentren in Deutschland geplant. https://www.zeit.de/news/ 2019-01/09/keine-weiteren-ankerzentren-in-deutschland-geplant-190109-99-485914. Zugegriffen: 9. Jan. 2019. 